\title{
Sensitivities in Large-Eddy Simulations of Mixed-Phase Arctic Stratocumulus Clouds Using a Simple Microphysics Approach*
}

\author{
COLLEen M. KAUL \\ Jet Propulsion Laboratory, California Institute of Technology, Pasadena, California, and Swiss Federal \\ Institute of Technology, Zurich, Switzerland
}

JOÃO TEIXEIRA

Jet Propulsion Laboratory, California Institute of Technology, Pasadena, California

KENTAROH SUZUKI

Atmosphere and Ocean Research Institute, University of Tokyo, Kashiwa, Japan

(Manuscript received 7 October 2014, in final form 17 June 2015)

\begin{abstract}
Arctic mixed-phase stratocumulus clouds are maintained by feedbacks between microphysical and dynamical phenomena, but the details of these interactions are incompletely understood. Although large-eddy simulations are a promising means of elucidating microphysics-turbulence relationships, the use of sophisticated microphysical schemes complicates analysis of their results. Here, the ability of a simplified one-moment scheme to capture basic features of this cloud type is investigated through simulations based on Mixed-Phase Arctic Cloud Experiment (MPACE), SHEBA/FIRE-ACE, and Indirect and Semi-Direct Aerosol Campaign (ISDAC) intercomparison studies. The results of the simple scheme show reasonable agreement with liquid and ice water path predictions reported by models using schemes of similar or greater complexity. Additional tests are performed to evaluate the sensitivity of the results to three main parameters of the scheme: the snow and ice size distribution intercept parameters and the exponent appearing in the temperature-dependent phasepartition function, which is used to diagnose cloud condensate amounts. Sensitivities of the SHEBA and ISDAC cases, both of which have low surface heat fluxes and low precipitation rates, tend to be similar, while the MPACE case, with higher surface fluxes and precipitation rates, shows somewhat different trends. Results of all three cases are found to be sensitive to the snow size distribution intercept parameter, but this quantity can be adequately estimated using a recently developed diagnostic expression based on observations of Arctic clouds.
\end{abstract}

\section{Introduction}

The importance of clouds to the global climate and the limited ability of current models to represent their effects have been widely noted. Marine stratocumulus clouds have particular importance in shaping the planetary energy budget owing to their extensive coverage and

\footnotetext{
* Supplemental information related to this paper is available at the Journals Online website: http://dx.doi.org/10.1175/MWR-D14-00319.s1.

Corresponding author address: Colleen M. Kaul, Geological Institute, Swiss Federal Institute of Technology Zurich, Soneggstrasse 5, 8092 Zurich, Switzerland.

E-mail: colleen.kaul@erdw.ethz.ch
}

strong radiative effects (e.g., Wood 2012). Stratiform clouds also appear to have a critical role in Arctic climate (Vavrus 2004) but differ in significant ways from their lower-latitude counterparts in terms of both their characteristics and their effects. Notably, Arctic clouds warm the surface over most of the year except for a brief midsummer period (Curry et al. 1996; Intrieri et al. 2002) and are implicated in variations in Arctic sea ice extent through cloud-radiation and surface-albedo feedbacks (Kay and Gettelman 2009; Vavrus et al. 2011).

One of the dominant features of Arctic cloud climatology is the prevalence of mixed-phase, low-level stratiform clouds (Shupe et al. 2006; Shupe 2011). Their presence is significant because the magnitudes of both longwave and shortwave cloud radiative forcings are greatly enhanced when the cloud composition includes 
some liquid (Shupe and Intrieri 2004), yet the frequent occurrence of such clouds is remarkable because of the inherent thermodynamic instability of supercooled liquid-ice mixtures (Morrison et al. 2012). Nevertheless, mixed-phase Arctic clouds have been observed to persist for several hours to several days (Shupe et al. 2006). A variety of sustaining feedbacks have been proposed to explain this longevity (Morrison et al. 2012).

Numerical modeling has already added greatly to understanding of Arctic mixed-phase stratocumulus clouds (AMPS), especially giving insight into how microphysical feedbacks may support their prolonged existence (e.g., Harrington et al. 1999; Jiang et al. 2000; Fridlind et al. 2007; Luo et al. 2008; Solomon et al. 2009; Ovchinnikov et al. 2011; Avramov et al. 2011). Nonetheless, at a basic level, cloud maintenance depends on maintaining enough moisture and sufficiently low temperatures to allow condensation to occur, which is not solely related to microphysical processes. For example, Solomon et al. (2011, 2014) have used simulations to investigate how AMPS maintain sufficiently high rates of moisture transport into the cloud layer to compensate for losses due to precipitation as well as to subsidence drying and dry air entrainment that occur in the absence of a moisture inversion. Gaining insight from ground-based observations of AMPS, Shupe et al. (2008) described how cloud-scale circulations can locally maintain clouds but they were not able to quantify the importance of the many potential drivers of vertical motion in AMPS. Clearly, detailed characterization of the structure of turbulence within AMPS is needed to fully understand how these clouds evolve.

Large-eddy simulation (LES) inherently provides threedimensional velocity and scalar fields with high spatial and temporal resolutions. Thus, LES models are theoretically well suited to the inclusion of highly sophisticated microphysical schemes, and increasing computational resources make the use of such models feasible. High-fidelity models offer the possibility of correctly predicting the details of a particular observed cloud without any ad hoc tuning, provided that model inputs (such as types and concentrations of ice nuclei) can be specified with sufficient accuracy. Nonetheless, it should be recalled that in the study of any complex system, a tension exists between "simulation" and "understanding" (Held 2005). Thus, there is value in retaining and refining simple modeling approaches even as ever more sophisticated models are developed. With a range of modeling options, a number of interrelated questions about the simulation of AMPS can be addressed, such as the following:

- How do different model components work together? Simulations are often designed in a highly modularized fashion, combining numerical schemes, turbulence closures, and microphysical models that may each have been developed in quite different contexts and tested under different conditions. With the many nonlinearities inherent in a turbulent, mixed-phase cloud, how these components come together to influence simulation outcomes cannot be taken for granted. It should be noted that even in liquid-only runs, LES participants in intercomparison studies of AMPS cases show a range of results (Klein et al. 2009; Ovchinnikov et al. 2014) that has not been clearly attributed to any specific difference in model formulation. Also, in addition to the difficulties of representing mixed-phase microphysics, the frequent occurrence of stable boundary layers in the Arctic poses a major challenge, since large-eddy simulations of stable boundary layers are known to be sensitive to grid resolution and subgrid turbulence closure (Beare et al. 2006).

- How can LES support improvements to the representation of AMPS in larger-scale models? Turbulent mixing takes place across a wide range of scales. Depending on model resolution, some scales of mixing can be explicitly simulated while smaller scales must be represented through closures. In contrast, microphysical schemes parameterize inherently small-scale phenomena. Depending on the temporal resolution of simulations, different approximations may be appropriate for computing formation and growth rates of hydrometeors. Nonetheless, none of these processes are resolved in the same way that LES or cloudresolving models necessarily resolve some scales of turbulent motion. Given these considerations, we can ask how predictions of AMPS (as well as other cloud types) differ between large-eddy simulations and coarser-resolution models in a series of steps. In this framework, a first step is to isolate the effects of modeled versus simulated turbulent mixing while using a simple microphysical scheme that is valid across a range of resolutions.

- What level of microphysical detail is needed to capture the response of AMPS to changes in largescale forcings? Considering AMPS as a component of the climate system, a primary question is how the radiative effects of AMPS change in response to changes to surface heat fluxes, large-scale moisture advection, and the like. The sensitivity of AMPS to such changes is not well understood (Morrison et al. 2012), and in fact the difficulties of specifying accurate large-scale forcings is a major hindrance in relating simulated and observed AMPS (e.g., Jiang et al. 2000). Different types of simulations can potentially be used to investigate these questions: from global models, which have two-way interaction between the large scales and (parameterized) small scales, to large-eddy 
simulations, in which large-scale forcings usually must be prescribed but clouds are resolved. An important question is how feedbacks between microphysical and dynamical processes either buffer or enhance the cloud response. A comprehensive answer requires that we evaluate the response across a range of scales and using various modeling options, again giving simple models a role alongside more complex ones.

With these applications for a simple, robust microphysical scheme in mind, the aim of this work is to develop a scheme that captures basic features of AMPS and depends on a limited number of parameters whose effects on simulation results can be comparatively easily characterized. For example, priority is placed on capturing the predominance of liquid water at cloud top, because it drives turbulence through radiative cooling. The scheme also seeks to reproduce the typical persistent precipitation of snow, which interacts with turbulence below the cloud base through the effects of vapor deposition and sublimation on buoyancy (Ovchinnikov et al. 2011).

The simple scheme is applied to the simulation of three different Arctic cloud cases, which were selected to represent the diverse conditions in which AMPS occur. All three cases are derived from observations and are therefore referred to by the name of the corresponding observational campaign: the Mixed-Phase Arctic Cloud Experiment (MPACE; Verlinde et al. 2007), the Indirect and Semi-Direct Aerosol Campaign (ISDAC; McFarquhar et al. 2011), and the Surface Heat Budget of the Arctic Ocean (SHEBA; Uttal et al. 2002) and First ISCCP Regional Experiment-Arctic Cloud Experiment (FIRE-ACE; Curry et al. 2000), which will be referred to simply as SHEBA. These cases have been the subjects of intercomparison studies (Klein et al. 2009; Morrison et al. 2011b; Ovchinnikov et al. 2014), which allows the performance of the new scheme to be readily put in context with the outcomes of existing approaches. This article presents the details of the scheme, describes the LES methodology and simulation setups, and discusses the sensitivities of the scheme to three main parameters that are related to the concentrations of cloud ice and snow and to the relative amounts of cloud liquid and ice.

\section{Microphysical modeling}

The proposed microphysical scheme is a one-moment bulk parameterization with diagnosed mixing ratios of cloud ice $q_{i}$ and cloud liquid $q_{l}$ and separate prognostic variables for mixing ratios of rain $q_{r}$ and snow $q_{s}$. Transformations between cloud condensate and precipitation categories are parameterized as autoconversion and aggregation source terms, which are explicitly computed as described in appendix A. Graupel, hail, and other categories of hydrometeors are not considered. Cloud condensate amounts are determined using a temperaturedependent, mixed-phase saturation adjustment procedure, similar to the approach used in the microphysical scheme by Grabowski (1998). In these types of approaches, a phase-partition function is used to adjust the local saturation vapor pressure between saturated values with respect to ice and to water, depending on the local temperature. The phase-partition function is again used to separate the total cloud condensate into cloud liquid and cloud ice components. Further details of the procedure are provided in appendix B. Since this is clearly an important component of the overall scheme, we discuss it first and explain our rationale for using such a simplified approach.

\section{a. Phase-partition function and diagnosis of cloud condensate}

Since the transition from liquid to ice critically affects cloud radiative properties and, therefore, cloud feedbacks (Li and Treut 1992; Gregory and Morris 1996), diagnostic relationships for cloud liquid fraction have long been of interest to climate researchers and there have been a number of efforts to characterize liquid-ice ratios in mixed-phase clouds using satellite (e.g., Devasthale and Thomas 2012) and in situ observations (e.g., Cober et al. 2001; Korolev et al. 2003; Boudala et al. 2004; McFarquhar et al. 2007; Noh et al. 2013). Specifically considering Arctic clouds, several studies have sought to understand the processes that control the relative proportions of liquid and ice (e.g., Rauber and Tokay 1991; Shupe et al. 2008; Korolev 2008; Shupe et al. 2006; Korolev and Isaac 2003; Korolev 2008; Morrison et al. 2005; Ervens et al. 2011; Avramov and Harrington 2010; Sulia and Harrington 2011). It should be noted that considerable uncertainty exists about the mechanisms of ice production in the atmosphere (Cantrell and Heymsfield 2005). Additionally, the properties of ice nuclei are often inadequately characterized, even when detailed measurements are made. Simulation results have been found to be sensitive to assumed concentrations and activation characteristics of ice nuclei, which are insufficiently constrained by observations (Fridlind et al. 2007, 2012).

Therefore, in LES that are not directly concerned with the details of ice initiation, it is arguably justified to use a simple parameterization to determine the phase partitioning of cloud condensate. Essentially, we would like the ability to investigate the consequences of imposed changes in phase partitioning on cloud dynamics without 
having to attempt to track all the changes in aerosol and ice nuclei populations and their associated ice initiation processes that underlie such changes in actual clouds. Therefore, a temperature-dependent expression for the liquid fraction $f_{l}$ of the form

$$
f_{l}= \begin{cases}1 & T \geq T_{\text {warm }} \\ 0 & T \leq T_{\text {cold }} \\ \left(\frac{T-T_{\text {cold }}}{T_{\text {warm }}-T_{\text {cold }}}\right)^{n} & \text { otherwise }\end{cases}
$$

is employed. The threshold temperatures $T_{\text {warm }}$ and $T_{\text {cold }}$ are set to 273 and $235 \mathrm{~K}$, respectively (DoutriauxBoucher and Quaas 2004; Boville et al. 2006). Expressions of this form have been proposed as phase-partition functions for large-scale (Doutriaux-Boucher and Quaas 2004; Boville et al. 2006; Grabowski 1998) and cloudresolving (Khairoutdinov and Randall 2003) models, all of which use $n \geq 1$. In some of the referenced models, the phase-partition function is used to determine the liquid fraction of both suspended and precipitating hydrometeors. That is, $f_{l}$ is used to determine the relative amounts of rain and snow as well as the relative amounts of cloud ice particles and cloud liquid droplets. In the present scheme, the phase-partition function is used only to diagnose the composition of cloud condensate. Rain and snow amounts are obtained by solving prognostic partial differential equations. Combining diagnostic and prognostic determinations of phase partitioning in this way helps to make the scheme more consistent with observations of phase partitioning in AMPS than purely diagnostic approaches to phase partitioning. Observationally derived profiles of liquid fraction that are computed considering all sizes of frozen particles commonly show regions in which the liquid fraction decreases with rising temperature owing to the growth of snow as it falls through the cloud (McFarquhar et al. 2007). This behavior is clearly not representable by Eq. (1) on its own but can be captured when snow amounts are tracked through a prognostic equation. Currently, $n$ is a free parameter of the scheme. We take $n=0.1$ as a baseline value since it assures $f_{l}$ greater than $80 \%$ over almost the entire range of mixed-phase conditions. This heavy predominance of the liquid phase in mixed-phase clouds is supported by observations (McFarquhar et al. 2007; de Boer et al. 2009).

It should be noted that the use of a diagnostic scheme for apportioning cloud condensate into ice and liquid categories is a major simplification that precludes an explicit calculation of the formation and growth processes affecting cloud droplet and cloud ice particles. In particular, the scheme lacks a mechanism to track the growth of cloud ice at the expense of cloud water when the environment is subsaturated with respect to ice but supersaturated with respect to liquid, a phenomenon often referred to as the Wegener-Bergeron-Findeisen mechanism [discussed, for example, by Morrison et al. (2012)]. Sedimentation of cloud ice and liquid droplets is also neglected. The effects of cloud liquid droplet sedimentation in simulations of AMPS have been discussed by Solomon et al. $(2011,2014)$ and Savre et al. (2014).

\section{b. Determination of particle size distribution function parameters}

The particle size distribution function (PSDF) of a hydrometeor species (denoted generically by subscript $x$ ) is presumed in the current scheme to follow an exponential distribution in the form

$$
N_{x}\left(D_{x}\right)=N_{0, x} \exp \left(-\lambda_{x} D_{x}\right) .
$$

This distribution has two parameters: $N_{0, x}$, referred to as the intercept parameter, and $\lambda_{x}$, referred to as the slope parameter. Particle diameter is denoted by $D_{x}$. Here, a subscript $x$ denotes any of the hydrometeor species: ice $(i)$, rain $(r)$, or snow $(s)$. Since only the mixing ratio of each hydrometer species is known, one of the parameters, usually the intercept parameter $N_{0, x}$, must be specified. Once this is done, $\lambda_{x}$ can be computed according to Eq. (A5).

Simulated AMPS have been shown to be sensitive to intercept parameter values, particularly $N_{0, s}$, and commonly assumed values for other regions and cloud types may not be applicable (Solomon et al. 2009; Luo et al. 2008). To attempt to overcome this limitation of onemoment schemes, a diagnostic relationship is used here for $N_{0, s}$. Such relationships usually depend on liquid or ice water content (Reisner et al. 1998) but may also depend on temperature (Thompson et al. 2004, 2008). Many of the relationships that have been proposed for $N_{0, s}$ are based on observations of midlatitude, deep frontal systems. Recently, however, a new relation has been developed using observations of shallow and deep frontal clouds in the Arctic (Morrison et al. 2011a), which is used here. Since precipitation in AMPS is predominantly snow, the rain intercept parameter, $N_{0, r}$, is expected to be much less important than $N_{0, s}$. Nonetheless, in the interest of reducing the number of free parameters in the scheme, a diagnostic relationship for $N_{0, r}$ is also included. Both of these diagnostic expressions are listed in appendix A. Difficulties in measuring small ice particles (e.g., McFarquhar et al. 2007) have impeded the formulation of empirical relations for the intercept parameter of the cloud ice PSDF, $N_{0, i}$. Therefore, an assumed value $N_{0, i}=1 \times 10^{7} \mathrm{~m}^{-4}$ is used 
as the baseline value. Explicit specification of the cloud liquid droplet PSDF is not required because information on droplet spectral shape is implicitly contained in an empirically based dispersion parameter, while particle number is determined by the assumed, fixed concentration of cloud condensation nuclei (see appendix A for additional information).

Although the use of a one-moment rather than twomoment formulation appears to be a significant limitation on the flexibility of the proposed scheme, it should be noted that prognosis of hydrometeor number concentrations involves its own difficulties. For instance, the lack of reliable data on small ice particle populations necessarily propagates uncertainty into predictions of snow particle populations. Solomon et al. (2009) found that 1-km-resolution simulations of MPACE using a two-moment scheme reasonably reproduced observed liquid and ice water contents and mean snow size spectra. However, when the total ice number concentration was increased to match observed values, liquid water was strongly depleted. Furthermore, various two-moment schemes make different assumptions about the way sublimation affects the population of snow particles, which feeds back to subsequent predictions of sublimation and sedimentation rates. Ovchinnikov et al. (2014) demonstrate the effect of this mechanism in large-eddy simulations of the ISDAC case, showing that predictions of particle mass mean diameter in the subcloud sublimation zone are closely tied to the assumed relation between changes in particle number and sublimation mass loss. Their results also show that two-moment schemes that follow either of the two most common assumed relationships fail to emulate the vertical profile of mean particle diameter produced by bin microphysical schemes. Nevertheless, this discussion is not meant to argue against the value of two-moment schemes but simply to point out that they are also affected by uncertainties. To the extent these uncertainties influence microphysical predictions in a more complex and subtle manner, a two-moment formulation is less advantageous than a one-moment formulation in applications where simplicity is prioritized.

\section{c. Precipitation source terms}

Four main microphysical processes affecting rain and snow mixing ratios are considered by the proposed scheme: autoconversion, accretion, deposition/sublimation (snow) or evaporation (rain), and sedimentation. Melting of snow is also included for completeness, although the three cases studied in this work are at sufficiently low temperatures to make this term inconsequential. Parameterizations for these processes have been taken from several sources. Details are provided in appendix A.

\section{Numerical discretization and simulation setup}

The large-eddy simulations presented in this work were performed by solving filtered anelastic flow equations on a staggered Cartesian mesh using a code known as the Jet Propulsion Laboratory Large-Eddy Simulation (JPLLES) model (Matheou et al. 2011). Prognostic variables include $u, v$, and $w$ velocities, total water mixing ratio $q_{t}$, rain mixing ratio $q_{r}$, snow mixing ratio $q_{s}$, and liquid-ice potential temperature $\theta_{\text {li }}$. The latter quantity is defined by Tripoli and Cotton (1981) as

$$
\theta_{\mathrm{li}}=\theta \exp \left(-\frac{L_{v} q_{l, \mathrm{tot}}}{c_{p} T}-\frac{L_{s} q_{i, \mathrm{tot}}}{c_{p} T}\right)
$$

where $\theta$ is potential temperature; $T$ is temperature; $L_{v}$ and $L_{s}$ are latent heats of vaporization and sublimation, respectively; and $c_{p}$ denotes specific heat. The variables $q_{l, \text { tot }}$ and $q_{i \text {,tot }}$ include the mixing ratios of all liquid and ice hydrometeors, whether suspended or precipitating. No latent heating terms are required in the prognostic equation for $\theta_{\mathrm{li}}$ because it is conserved during changes of phase, but it is necessary to account for the differential motion of precipitating hydrometeors.

A transport equation for the subgrid turbulent kinetic energy $e$ is included as part of a 1.5-order turbulence closure (Klemp and Wilhemson 1978; Deardorff 1980). In particular, with $l$ denoting the buoyancy-limited mixing length $l \leq(\Delta x \Delta y \Delta z)^{1 / 3}$ as given by Eq. (10a) of Deardorff (1980), the eddy viscosity $v_{T}$ and eddy diffusivity $D_{T}$ are diagnosed from

$$
\begin{aligned}
v_{T} & =c_{k} e^{1 / 2} l \text { and } \\
D_{T} & =\left[1+2 \frac{l}{(\Delta x \Delta y \Delta z)^{1 / 3}}\right] v_{T}
\end{aligned}
$$

and the dissipation rate $\varepsilon$ of turbulent kinetic energy is found from

$$
\varepsilon=\left[1.9 c_{k}+\left(0.93-1.9 c_{k}\right) \frac{l}{(\Delta x \Delta y \Delta z)^{1 / 3}}\right] \frac{e^{3 / 2}}{l} .
$$

All simulations shown here use the value $c_{k}=0.1$.

Radiative fluxes are computed using the subroutine BUGSRAD, where BUGS is the name of an atmospheric general circulation model developed at Colorado State University (Stephens et al. 2001). BUGSRAD considers 6 shortwave and 12 longwave spectral bands. Surface albedo is specified as 0.827 for SHEBA simulations (Morrison et al. 2011b) and is taken to be 0.1 and 0.8 in the MPACE and ISDAC simulations, respectively, consistent with their open-ocean and ice-covered surface 
conditions. To obtain downwelling radiative fluxes, thermodynamic profiles in each column of the LES domains (which have heights of only a few kilometers) are smoothly patched to specified profile data. Since these profile data are not included in the published intercomparison references, but rather were provided via websites, they have been included in the online supplemental material accompanying this publication.

A second-order accurate spatial discretization is used for advection of momentum and all diffusion terms as described by Matheou et al. (2011). Scalar advection terms are evaluated using a fifth-order weighted essentially nonoscillatory (WENO) scheme to provide a highaccuracy, nonoscillatory discretization (Jiang and Peng 2000).

Temporal advancement is performed using the thirdorder Runge-Kutta method of A. Wray as presented in Spalart et al. (1991). The length of the time step is dynamically adjusted to maintain a Courant number no greater than 0.8. Each Runge-Kutta substep begins with the diagnosis of temperature $q_{i}$ and $q_{l}$ from the prognosed values of $\theta_{\mathrm{li}}, q_{t}, q_{r}$, and $q_{s}$. This is done using a Newton-Raphson technique described in appendix B. Once the thermodynamic update is accomplished, microphysical tendencies of $q_{r}$ and $q_{s}$ are determined using the source term equations specified in appendix A. Tendencies of $q_{r}$ and $q_{s}$ due to sedimentation are also calculated; these imply tendencies of $q_{t}$ and $\theta_{\mathrm{li}}$ as well. The substep proceeds with the computation of tendencies due to advection, subgrid-scale mixing, radiation, and large-scale forcing, following standard methods for large-eddy simulation codes.

These simulations use modest resolutions and domain sizes to allow a number of runs to be performed with varying microphysical parameters. Information on grid resolution and domain size is summarized in Table 1. Additional runs with larger domains were performed over a subset of the parameter space for shorter simulation periods of $12-18 \mathrm{~h}$. Doubling, and then quadrupling, the domain size produced no significant changes in the results of simulations of the SHEBA and ISDAC cases. In contrast, MPACE results showed dependence on horizontal domain size but were not very sensitive to horizontal resolution. Therefore, horizontal domain extents up to $40 \mathrm{~km} \times 40 \mathrm{~km}$ were tested by increasing the horizontal grid spacing to $80 \mathrm{~m}$. Although not totally domain-size independent, the sensitivity of the results significantly decreased for horizontal domain extents of $10 \mathrm{~km}$ and larger. Therefore, this domain size is used for the MPACE simulations shown here.

The simulation setups of MPACE, SHEBA, and ISDAC cases follow the specifications of their respective intercomparison studies (Klein et al. 2009; Morrison
TABLE 1. Grid resolutions and number of grid points used for simulations of each case.

\begin{tabular}{ccccc}
\hline \hline Case & $\Delta x, \Delta y(\mathrm{~m})$ & $\Delta z(\mathrm{~m})$ & $N_{x}, N_{y}$ & $N_{z}$ \\
\hline ISDAC & 40 & 20 & 64 & 196 \\
SHEBA & 40 & 10 & 64 & 250 \\
MPACE & 80 & 20 & 128 & 196 \\
\hline
\end{tabular}

et al. 2011b; Ovchinnikov et al. 2014), except where they involve a modification of the microphysical or radiation scheme. An additional minor modification to the largescale forcing applied to the ISDAC case simulations is described in section 4 a.

\section{Results}

The results of simulations using the microphysical scheme are presented in three parts. First, key features of each case are described and illustrated using the results of baseline simulations with a fixed set of simulation parameters: $N_{0, s}$ determined from the diagnostic relationship of Morrison et al. (2011a), $n=0.1$ (see section 2a), and $N_{0, i}=1 \times 10^{7} \mathrm{~m}^{-4}$. This value of $N_{0, i}$ is selected as intermediate between the assumed and predicted values of $N_{0, i}$ used by Solomon et al. (2009) for MPACE simulations. Second, liquid water path (LWP) and ice water path (IWP) predictions of the baseline simulations are compared to the values obtained in past model intercomparison studies. Finally, the sensitivities of the results to the specifications of $N_{0, s}, N_{0, i}$, and $n$ are explored.

In our discussion, we refer to various regions in the boundary layer. Although these regions are mainly referenced in a qualitative context, we briefly review our terminology here. First, the term boundary layer refers to the extent of the atmosphere between the surface and the temperature inversion. Since the temperature inversion is strong in all of the cases simulated here, the boundary layer height can be readily identified. By mixed layer, we indicate any portion of the boundary layer where the vertical gradient in liquid-ice potential temperature is approximately zero. Quantitatively, the cloud top and cloud base are identified with the maximum and minimum average height for which $q_{l}>0.01 \mathrm{~g} \mathrm{~kg}^{-1}$ or $q_{i}>0.0001 \mathrm{~g} \mathrm{~kg}^{-1}$ (Klein et al. 2009). The cloud deck is the portion of the boundary layer between the cloud top and cloud base, while subcloud refers to the region below the cloud base.

\section{a. Description of cases}

\section{1) MPACE}

MPACE was conducted near Barrow, Alaska, with the purpose of gathering detailed measurements of 

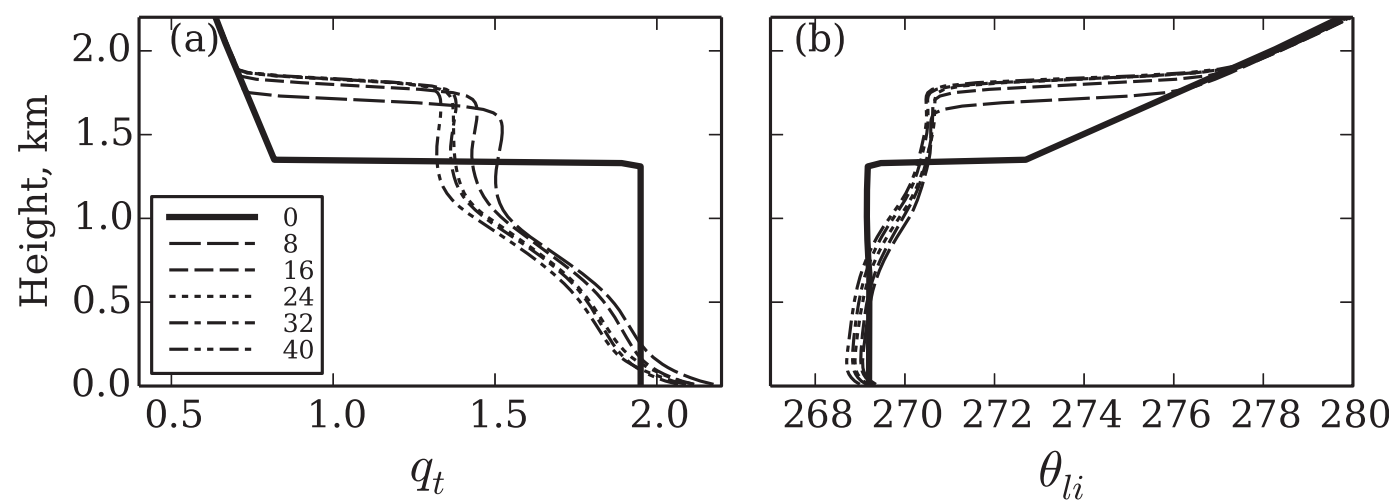

FIG. 1. Mean profiles from MPACE baseline simulation of (a) total water mixing ratio $q_{t}$ in $\mathrm{g} \mathrm{kg}^{-1}$ and (b) liquidice potential temperature $\theta_{\mathrm{li}}$ in $\mathrm{K}$. Profiles are averaged over 1-h windows centered at the times shown in the figure legend, except for the initial condition indicated by the heavy, solid line.

mixed-phase Arctic clouds during the autumnal transition season. A mixed-phase, single-layer stratocumulus was observed during the period 9-11 October 2004 as air flowed off the pack ice over open ocean (Verlinde et al. 2007). McFarquhar et al. (2007) describe macro- and microphysical characteristics of the cloud as measured during research flights, while Shupe et al. (2008) analyze cloud properties using ground-based instruments and van Diedenhoven et al. (2009) compare cloud radar and lidar measurements to synthetic observations derived from large-eddy simulations by Fridlind et al. (2007). The observed cloud of 9-11 October was subsequently used as the basis for a model intercomparison study (Klein et al. 2009).

In the intercomparison scenario, which has also been used for several other computational studies (e.g., Avramov and Harrington 2010; Luo et al. 2008), an initially well-mixed boundary layer with a depth of approximately $1300 \mathrm{~m}$ is driven by strong cloud-top radiative cooling and surface sensible and latent heat fluxes as well as significant shear. The boundary layer is capped by a temperature inversion of approximately $2 \mathrm{~K}$ and the cloud-top temperature is approximately $258 \mathrm{~K}$.

Profiles of thermodynamic variables are plotted in Fig. 1. As described above, the initial profiles are well mixed. At $8 \mathrm{~h}$ (long dashed lines), distinct layers associated with the cloud deck and subcloud region are evident and the boundary layer height has increased by a few hundred meters. This pattern is maintained for the remainder of the simulations. Similarly, an initial adjustment period, followed by the attainment of quasisteady statistics is also apparent in mean profiles of $q_{l}$ and $q_{s}$ plotted in Fig. 2. These quantities both show a pattern of fluctuating values with a period of roughly $2 \mathrm{~h}$. Peaks in cloud liquid content appear to slightly lead peaks in snow content and the highest values of $q_{s}$ are obtained at or below cloud base, suggesting that snow continues to grow through vapor deposition as it falls.

Examination of snow tendencies (Fig. 3) confirms the importance of vapor deposition for increasing snow amounts. Figure 3 shows mean profiles of tendencies averaged over hours 12 and 36 of the simulation. Except at the very top of the cloud, deposition/sublimation and transport are the largest tendencies and are opposite in sign. Autoconversion rates peak near cloud top, while accretion acts as a source of snow through the depth of the cloud. Figure $3 c$ shows the integrated values of the source terms through the depth of the boundary layer. The integrated source term of autoconversion varies smoothly with time and quickly plateaus to a nearconstant value. However, the other terms fluctuate strongly, especially the deposition/sublimation term, which varies from being a net source to a net sink of snow mass. Interestingly, positive fluctuations in the aggregation rate occur slightly before positive fluctuations in deposition, which mirrors the pattern in the fluctuations of $q_{l}$ and $q_{s}$ noted in Fig. 2. These results indicate a cycle in which positive fluctuations in the amount of cloud condensate leads to enhanced snow production. As the snow sediments, it grows through aggregation and then, more strongly, through deposition. These processes deplete the positive fluctuation in cloud condensate. Finally, a large portion of the snow sublimates before reaching the surface, generating negatively buoyant air through sublimation cooling that suppresses vertical motion in the vicinity. For more discussion on this topic, please see section 4c.

Based on the results shown in Figs. 2 and 3, we can explain the transition away from the initial well-mixed boundary layer structure that is depicted in Fig. 1. Sedimentation of snow dries and warms the cloud layer. Cloud-top radiative cooling also drives strong local 

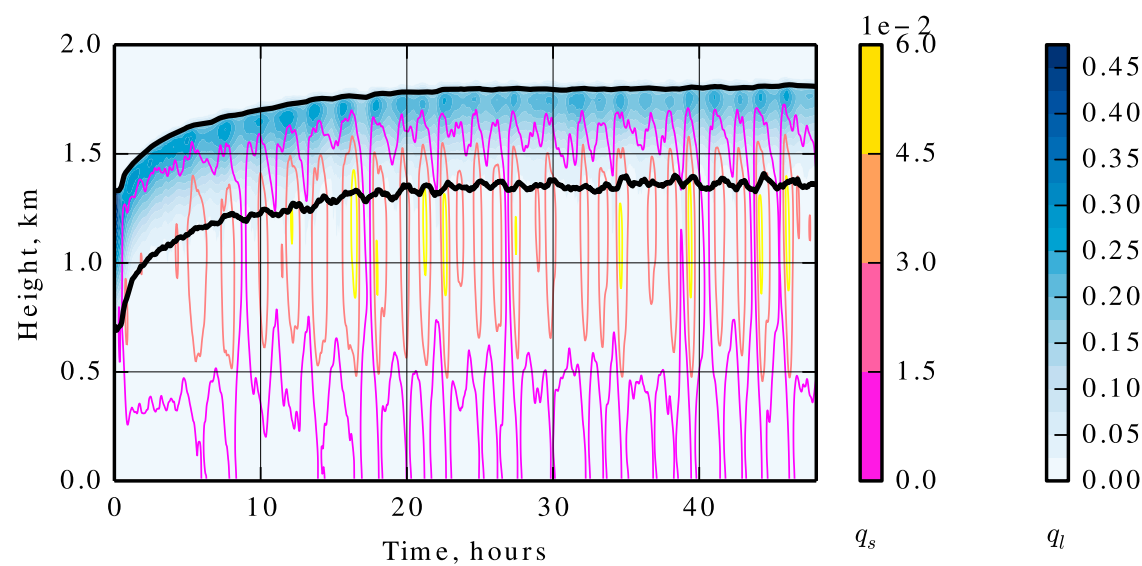

FIG. 2. Profiles of mean cloud liquid mixing ratio $q_{l}$ shown as blue colored shading and overlaid by colored contour lines of mean snow mixing ratio $q_{s}$ from MPACE baseline simulation. Mixing ratio values are given in $\mathrm{g} \mathrm{kg}^{-1}$. Solid black lines show cloud-top and cloud-base height according to the definition given in section 4 .

turbulence that mixes in warm, dry air from above the inversion (not shown). Snow sublimation below the cloud has a moistening and cooling effect. Although large heat fluxes and shear help to produce surfacedriven turbulence, mixing is not sufficiently vigorous to eliminate the two-layer structure seen in Fig. 1.

\section{2) SHEBA/FIRE-ACE}

A persistent low-level, mixed-phase cloud was observed in the vicinity of the SHEBA (Uttal et al. 2002) site during the period 1-10 May 1998. Aircraft measurements were made of this cloud on 4 and 7 May as part of FIRE-ACE (Curry et al. 2000) and analyzed in detail by Zuidema et al. (2005).
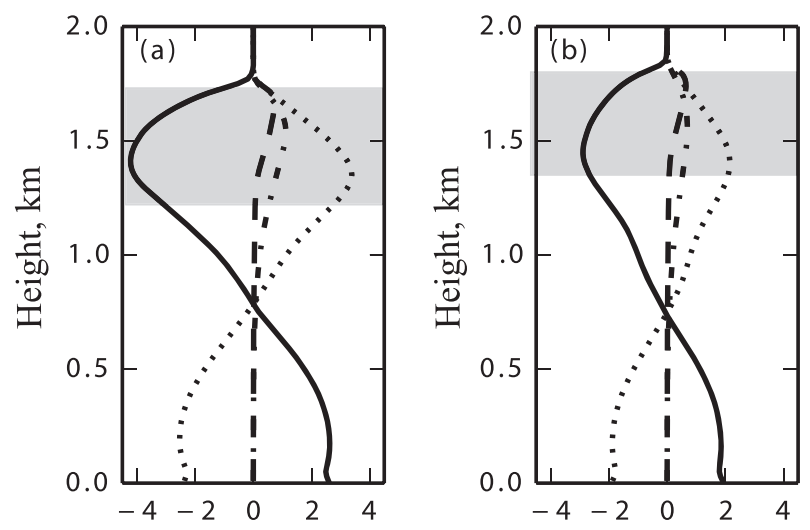

Tendency, $\mathrm{g} \mathrm{kg}^{-1} \mathrm{~d}^{-1}$
Data from the 7 May research flight were subsequently used as the basis for a large-eddy simulation and cloudresolving model intercomparison study by Morrison et al. (2011b). The setup of our SHEBA simulations closely follows the specifications of the SHEBA intercomparison, except that it does not apply the modified ice nucleation rate expression that was used to constrain the ice particle number density predictions of models participating in the intercomparison. As in MPACE, the boundary layer is initially specified as well mixed, but is considerably shallower with much weaker surface heat fluxes. Additionally, temperatures at the surface and within the cloud are around $10 \mathrm{~K}$ cooler than in MPACE, and the cloud is capped by a stronger temperature inversion of $5-6 \mathrm{~K}$.

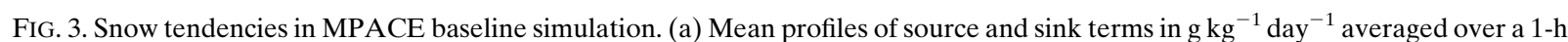
window centered at hour 12. Gray shading indicates the portion of the profile between the average cloud top and cloud base. (b) As in (a), but at hour 36. (c) Source and sink terms in $\mathrm{g} \mathrm{m}^{-2} \mathrm{~h}^{-1}$ integrated over boundary layer depth . Quantities plotted are transport (TRAN, solid) including sedimentation, resolved mixing and subgrid-scale mixing, autoconversion (AUT, dashed), accretion (ACC, dot-dashed) including snow-liquid, snow-ice, and snow-rain collisions, and vapor deposition and sublimation (D/S, dotted). 

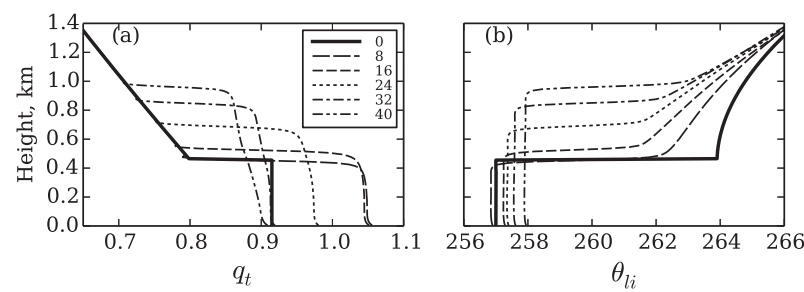

FIG. 4. As in Fig. 1, but from SHEBA.

Mean profiles of $q_{t}$ and $\theta_{\mathrm{li}}$ are plotted in Fig. 4 . Through the first $8 \mathrm{~h}$ (long dashed lines), the boundary layer height remains nearly unchanged, while the mixed layer moistens and slightly cools. Subsequently, the boundary layer deepens and the mixed-layer value of $q_{t}$ decreases while that of $\theta_{\mathrm{li}}$ increases. The evolution of cloud-top and cloud-base heights is shown clearly in Fig. 5, which plots mixing ratios of cloud liquid and snow. As the boundary layer moistens over the first several hours, the cloud base falls and $q_{l}$ values increase. Once the cloud top begins to rise after about $10 \mathrm{~h}$, cloud base ascends along with it. Peak values of $q_{l}$ continue to increase and snow amounts grow. Again, maximum snow amounts are found at or below the cloud base, suggesting depositional growth is important.

Figure 6 shows source and sink terms of snow. Unlike the MPACE case, aggregation processes make a negligible contribution to the amount of snow, a result that is substantiated by ice particle imagery that shows a preponderance of crystal types with low aggregation efficiency (Morrison et al. 2011a). Additionally, the magnitudes of all source terms are much smaller, and their fluctuations are low. Autoconversion and vapor deposition onto snow are the dominant source terms, and the column-integrated magnitude of each of these terms grows with time. Through hour 12 of the simulation, the mean source term profiles show only depositional growth (Fig. 6a). Subsequently, as the boundary layer deepens, warms, and dries, the mean profiles show sublimation losses occurring above the surface in a layer whose depth increases with time (Fig. 6b). Despite increasing amounts of sublimation, the net contribution of depositional growth increases. By the latter half of the simulation, the autoconversion and snow depositional growth terms contribute nearly equally to the snow mass budget (Fig. 6c).

\section{3) ISDAC}

ISDAC, conducted during April 2008 near Barrow, Alaska, sought to characterize springtime Arctic clouds and compare them to autumnal clouds, such as those observed during MPACE (McFarquhar et al. 2011). Measurements of a single-layer stratocumulus cloud deck on 26 April have been used as the basis for a largeeddy simulation intercomparison (Ovchinnikov et al. 2014) as well as several earlier computational studies (Fan et al. 2011; Ovchinnikov et al. 2011; Yang et al. 2013; Hill et al. 2014).

In contrast to the SHEBA and MPACE cases, the boundary layer in the ISDAC 26 April case is decoupled, with a stable layer extending $400 \mathrm{~m}$ from the surface to the base of a cloud-topped, well-mixed layer. Because the boundary layer is decoupled, heat and moisture fluxes from the ice-covered surface are neglected in the intercomparison specification (Ovchinnikov et al. 2014), and we follow suit here. The large-scale subsidence forcing of the intercomparison is also replicated in our simulations. However, an alternative treatment is used for large-scale advective forcing. Rather than nudge the mean scalar profiles toward their initial values, explicit forcing terms are used to allow simulations to evolve with less constraint. These forcing terms are constructed to balance the effects of subsidence on the initial profiles
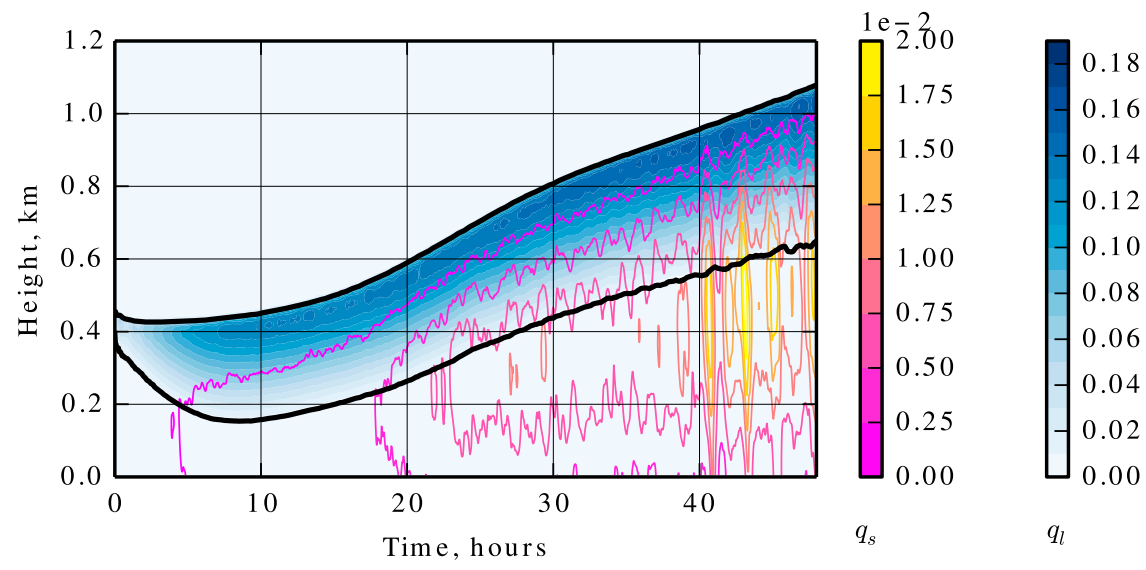

FIG. 5. As in Fig. 2, but from SHEBA. 

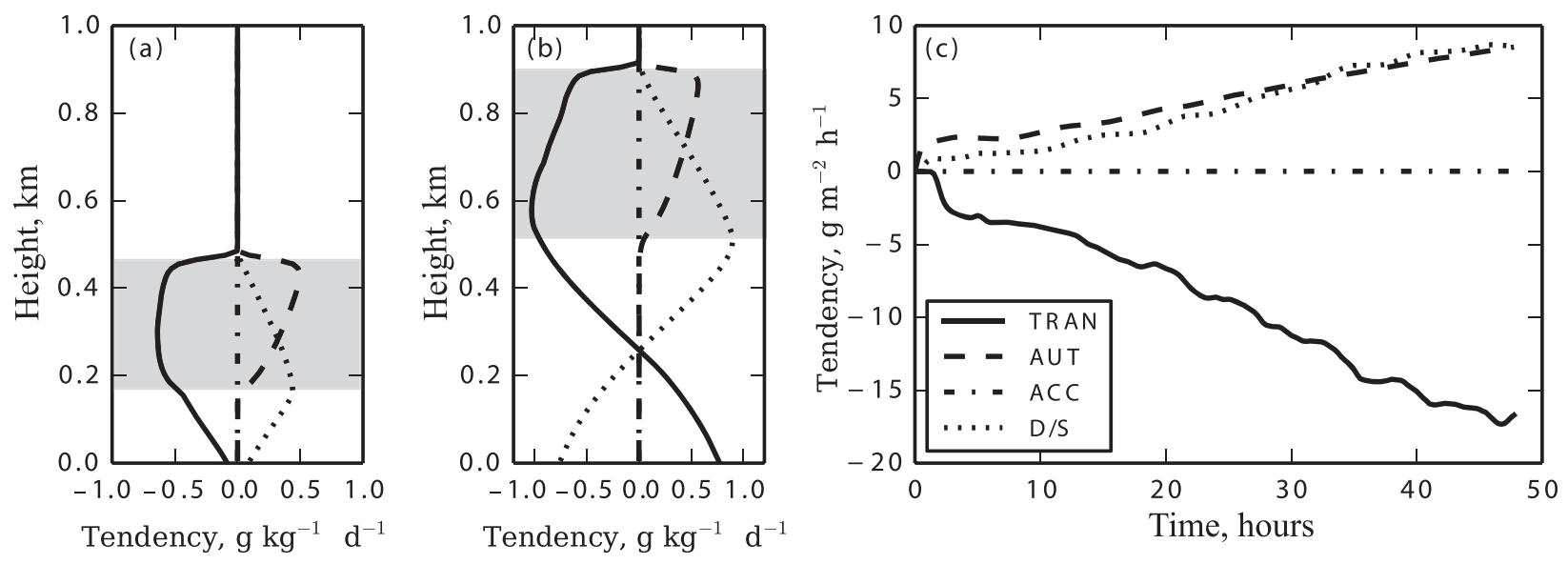

FIG. 6. As in Fig. 3, but from SHEBA.

and to compensate for radiative cooling of $1 \mathrm{~K} \mathrm{day}^{-1}$ above the boundary layer. It should be noted that the large-scale forcing is weak compared to the other cases simulated here, especially the SHEBA case. Although little or no moisture is supplied by the surface fluxes or large-scale forcing, the stable layer in the ISDAC case has higher water content than the well-mixed layer above and therefore is a potential source of moisture for the cloud.

Figure 7 plots mean profiles of $q_{t}$ and $\theta_{\mathrm{li}}$. The initial profiles clearly show a moist, stable layer rising from the surface to the cloud-topped mixed layer above. This stable layer is eroded as the simulation proceeds, blending with the mixed layer. Boundary layer depth increases, and the profiles dry and cool. This cooling is attributable to longwave radiation and helps to sustain the cloud despite the drying tendency of precipitation. Profiles of cloud liquid and snow mixing ratios are plotted in Fig. 8. The high-frequency variations of $q_{l}$ and $q_{s}$ found in MPACE and at the end of the SHEBA simulations are not apparent in these results; however, unlike those other cases, $q_{l}$ and $q_{s}$ vary diurnally, with maxima occurring near midnight (hours 14 and 38 of the simulation) and minima occurring near noon (hours 2 and 26). Of the three cases, the ISDAC case has the largest diurnal variation in incoming solar radiation. Moreover, simulations are sensitive to the rate of radiative cooling, which helps to compensate for drying due to precipitation as mentioned above. The cloud base descends during the night and ascends during the day, while gradually rising overall. Cloud tops ascend throughout the simulation, although the rate of ascent is more rapid overnight.

Tendencies of snow are plotted in Fig. 9. Like snow tendencies from SHEBA, the magnitudes of the source terms are smaller than in MPACE and show low temporal fluctuations. A notable feature, and key difference from SHEBA, is that deposition and sublimation tendencies are approximately balanced so that the net depositional source of snow is small. Therefore, much of the snow that sediments from the cloud never falls to the surface but rather returns to the vapor phase and can potentially be transported back into the cloud. This helps to explain how the cloud can be maintained over many hours despite the absence of significant moisture sources from surface fluxes and large-scale advection.

\section{b. Comparison to intercomparison results}

Figure 10 plots time series of liquid and ice water path statistics from the three cases. Here, ice water path includes contributions from cloud ice as well as snow. These statistics can be compared to LWP and IWP values reported from intercomparison results and observations to verify that the new scheme gives reasonable results.

Time series of liquid and ice water path of the MPACE simulation are plotted in Fig. 10a. LWP falls rapidly over the first few hours of the simulation as IWP rises. However, after roughly 10 to 15 hours, these quantities plateau to a nearly constant mean value that is maintained for the remainder of the simulation. In

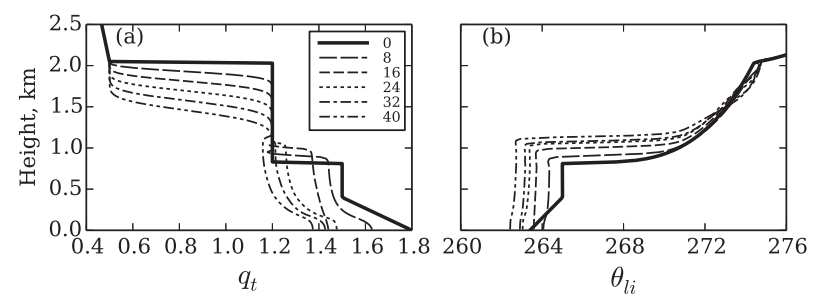

FIG. 7. As in Fig. 1, but from ISDAC. 

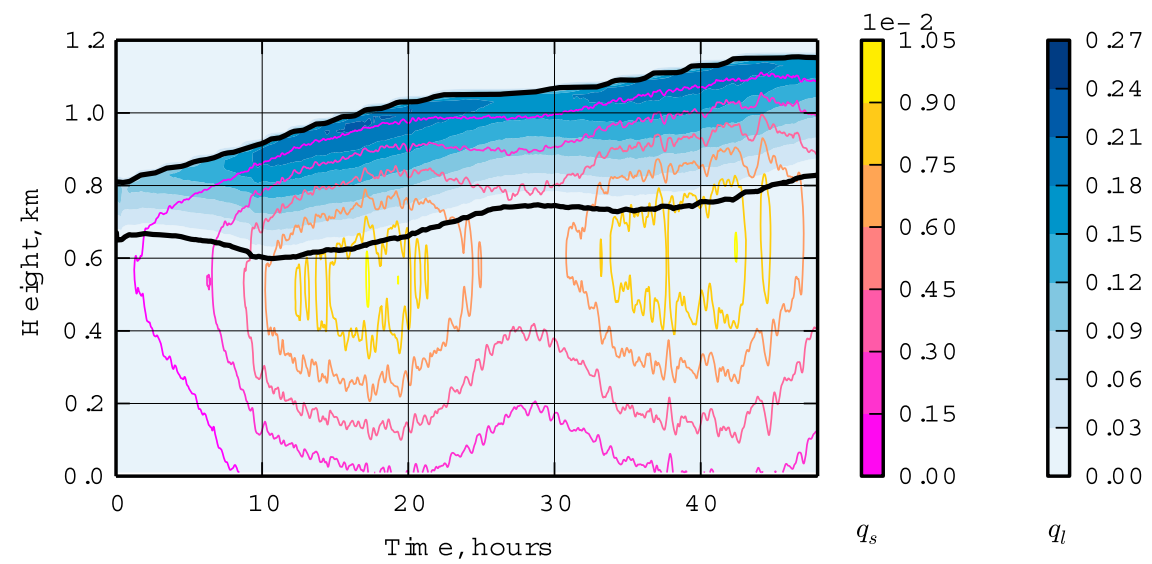

FIG. 8. As in Fig. 2, but from ISDAC.

contrast to the behavior shown by the other two cases, rapid variations are superimposed on the steady LWP and IWP trends. All time series statistics are sampled at 100 -s intervals, indicating that the noisy appearance of the fluctuations is not an artifact of a low sampling rate. A mechanism to explain these fluctuations is proposed in section 4c.

Values of liquid and ice water path measured during the MPACE observational period show significant differences depending on their spatial location and the instrument used, whether ground based or aircraft mounted, and estimates of LWP and IWP range, respectively, between $110-210$ and $8-30 \mathrm{~g} \mathrm{~m}^{-2}$ (Klein et al. 2009). The MPACE intercomparison study by Klein et al. (2009) compares models based on median values of LWP and IWP computed over the final 8 hours of 12-h simulations. For our simulation, these median values are $84 \mathrm{~g} \mathrm{~m}^{-2}$ for LWP and $42 \mathrm{~g} \mathrm{~m}^{-2}$ for IWP, thus underpredicting LWP and overpredicting IWP relative to the observationally based estimates. However, it should be recalled that the simulations use idealized forcings and surface fluxes that are not precisely matched to actual conditions. With this in mind, the more relevant comparison is between our simulation and the model data provided in Table IV of Klein et al. (2009). According to these data, the three LES participants using bulk microphysical schemes yield median LWP between 23 and $30 \mathrm{~g} \mathrm{~m}^{-2}$ (red box in Fig. 10a) and median IWP ranging from 22 to $34 \mathrm{~g} \mathrm{~m}^{-2}$ (blue box in Fig. 10a), showing a large quantitative error in LWP and significant qualitative error in the relative proportions of liquid and ice. Considering all model types (one, two, and three dimensional), our simulation's IWP values are higher than the median value of $32 \mathrm{~g} \mathrm{~m}^{-2}$ for single-moment schemes (blue line) and $20 \mathrm{~g} \mathrm{~m}^{-2}$ for double-moment schemes, with most of the ice water content contributed by snow. Nevertheless, the LWP predictions of the new scheme are comparable to the median predictions of

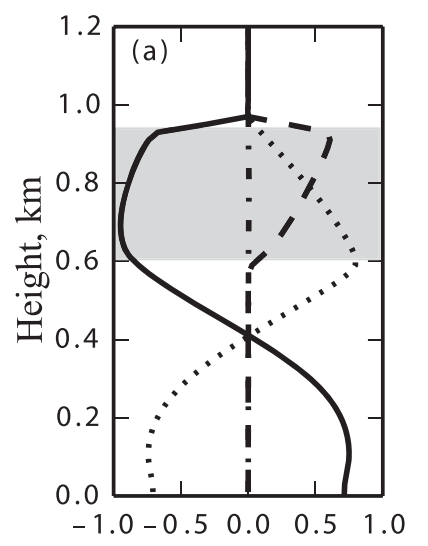

Tendency, $\mathrm{g} \mathrm{kg}^{-1} \mathrm{~d}^{-1}$

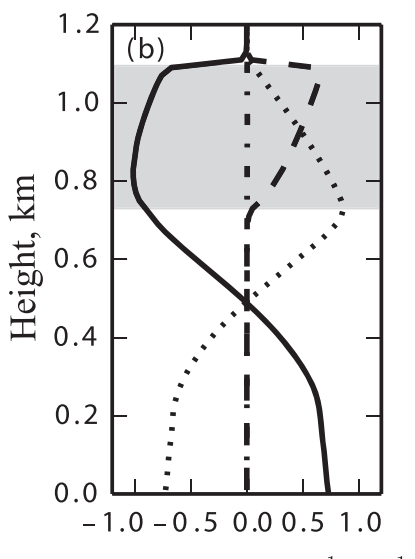

Tendency, $\mathrm{g} \mathrm{kg}^{-1} \mathrm{~d}^{-1}$

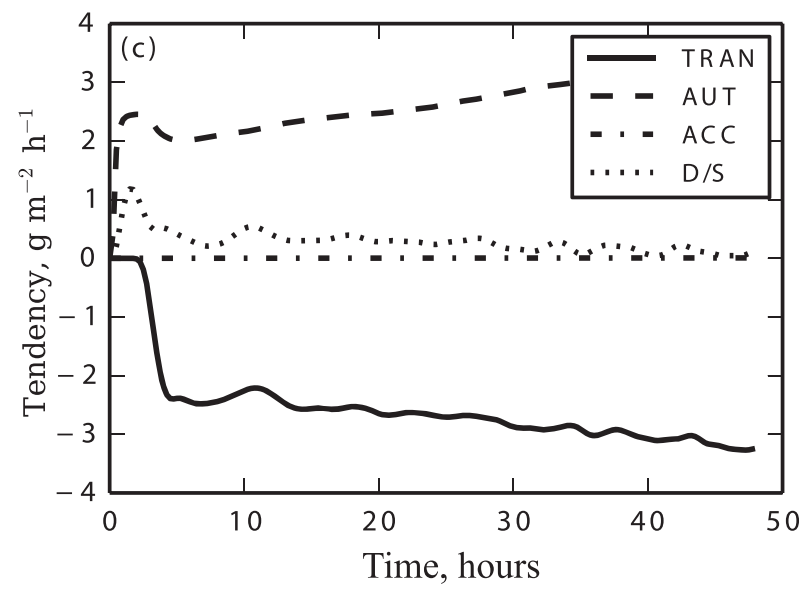

FIG. 9. As in Fig. 3, but from ISDAC. 

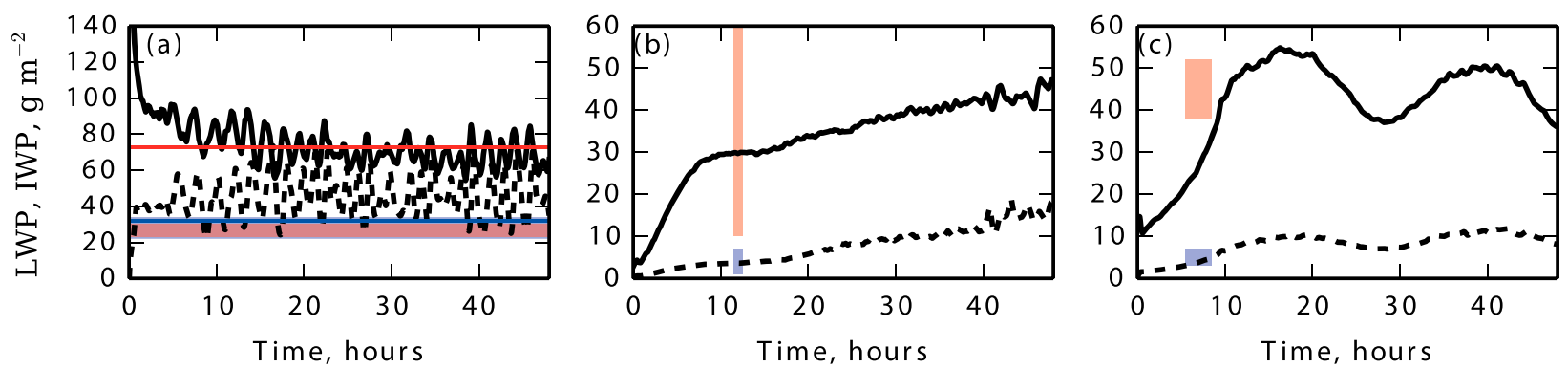

FIG. 10. Time series of liquid water path (solid) and total ice water path (dashed), which includes both cloud ice and snow from baseline simulations of (a) MPACE, (b) SHEBA, and (c) ISDAC. Colored straight lines and rectangular boxes indicate values of LWP (red) and IWP (blue) predicted by intercomparison ensembles. Detailed explanation is provided in the text.

single- and double-moment schemes, which are $73 \mathrm{~g} \mathrm{~m}^{-2}$ (red line) and $100 \mathrm{~g} \mathrm{~m}^{-2}$, respectively.

SHEBA time series show two periods of development (see Fig. 10b). The first period extends over approximately the first 8 hours of the simulation, while the second period extends over its remainder. LWP increases rapidly over the first period, but thereafter, during the second period, LWP increases at a steady, more gradual rate. This is consistent with the evolution of $q_{l}$ and $q_{s}$ shown in Fig. 5. The concentrations of ice particles predicted by the scheme over the first 12 simulation hours (see Fig. C2h in appendix C) are comparable to the target ice concentration of $1.7 \mathrm{~L}^{-1}$ in the "baseline" runs of the SHEBA intercomparison study by Morrison et al. (2011b). Despite the constrained treatment of ice concentrations, model predictions vary widely in the intercomparison's baseline runs, with one model predicting complete glaciation of the cloud within $2 \mathrm{~h}$ and another yielding steadily increasing LWP values that reach $60 \mathrm{~g} \mathrm{~m}^{-2}$ by the end of the 12 -h simulations. Simulation-end LWP values from the remaining four intercomparison participants range from 10 to $20 \mathrm{~g} \mathrm{~m}^{-2}$. At the same time, IWP predictions vary between 1 and $7 \mathrm{~g} \mathrm{~m}^{-2}$. These ranges are indicated by red and blue boxes, respectively, in Fig. 10b. Our scheme predicts LWP of $30 \mathrm{~g} \mathrm{~m}^{-2}$ and IWP of $3.6 \mathrm{~g} \mathrm{~m}^{-2}$ at hour 12 , and thus once again the results are found to be reasonably consistent with those obtained from other schemes, especially given the large spread in their results.

The ISDAC intercomparison by Ovchinnikov et al. (2014) considered two sets of mixed-phase simulations with target ice concentrations of 1 and $4 \mathrm{~L}^{-1}$ (designated ice1 and ice4, respectively). Additionally, other aspects of the ice microphysics, including mass-diameter, diameter-capacitance, and diameter-velocity relations, were specified to minimize sources of discrepancy among the participating models. Nevertheless, at the end of the 8-h intercomparison simulations the models showed substantial spread in their results, particularly at higher ice concentrations. LWP predictions of the intercomparisons ranged from approximately 38 to $52 \mathrm{~g} \mathrm{~m}^{-2}$ for ice 1 runs (indicated by red box in Fig. 10c) and from 12 to $38 \mathrm{~g} \mathrm{~m}^{-2}$ for ice 4 runs. IWP predictions ranged between approximately 3 and $7 \mathrm{~g} \mathrm{~m}^{-2}$ for ice 1 runs (indicated by blue box) and between 8 and $20 \mathrm{~g} \mathrm{~m}^{-2}$ for ice 4 runs. The width of the boxes (between hours 6 and 8 of our simulations) is used to indicate the uncertainty in matching the timing of our simulations and those of the intercomparison because we did not include an ice-free spinup period. Although the ice1 runs use an ice concentration that is estimated from aircraft-based measurements (McFarquhar et al. 2011), all models predict LWP values higher than those obtained from ground-based observations. Observed LWP during the period vary from $<10$ to $20 \mathrm{~g} \mathrm{~m}^{-2}$ depending on the retrieval algorithm used (Liu et al. 2011). Ice number concentrations were not explicitly constrained in our microphysical scheme as doing so would not permit the use of the diagnostic relationship for $N_{0, s}$, which is expected to be an important component of the new scheme. Additionally, ice particle size, velocity, and capacitance relations were not modified from those given in appendix A. Using the baseline parameter set of $N_{0, i}=1 \times 10^{7} \mathrm{~m}^{-4}, n=0.1$, total number concentrations of ice (including cloud ice and snow) have values of approximately $2 \mathrm{~L}^{-1}$ within the cloud and $1 \mathrm{~L}^{-1}$ below the cloud base (see Fig. C2i in appendix C). Our simulation predicts LWP and IWP values of 31 and $4.5 \mathrm{~g} \mathrm{~m}^{-2}$, respectively, at hour 8 . Our IWP value is in the range of intercomparison results for the ice1 simulations, while the LWP is somewhat low, as would be expected from the higher ice concentrations actually obtained in our simulations. It should be noted that the intercomparison simulations did not include the effects of solar radiation, although diurnal variation appears in these results. However, this appears to be of limited significance to the increase in LWP and IWP occurring over the first several hours that is driven by mixing of 

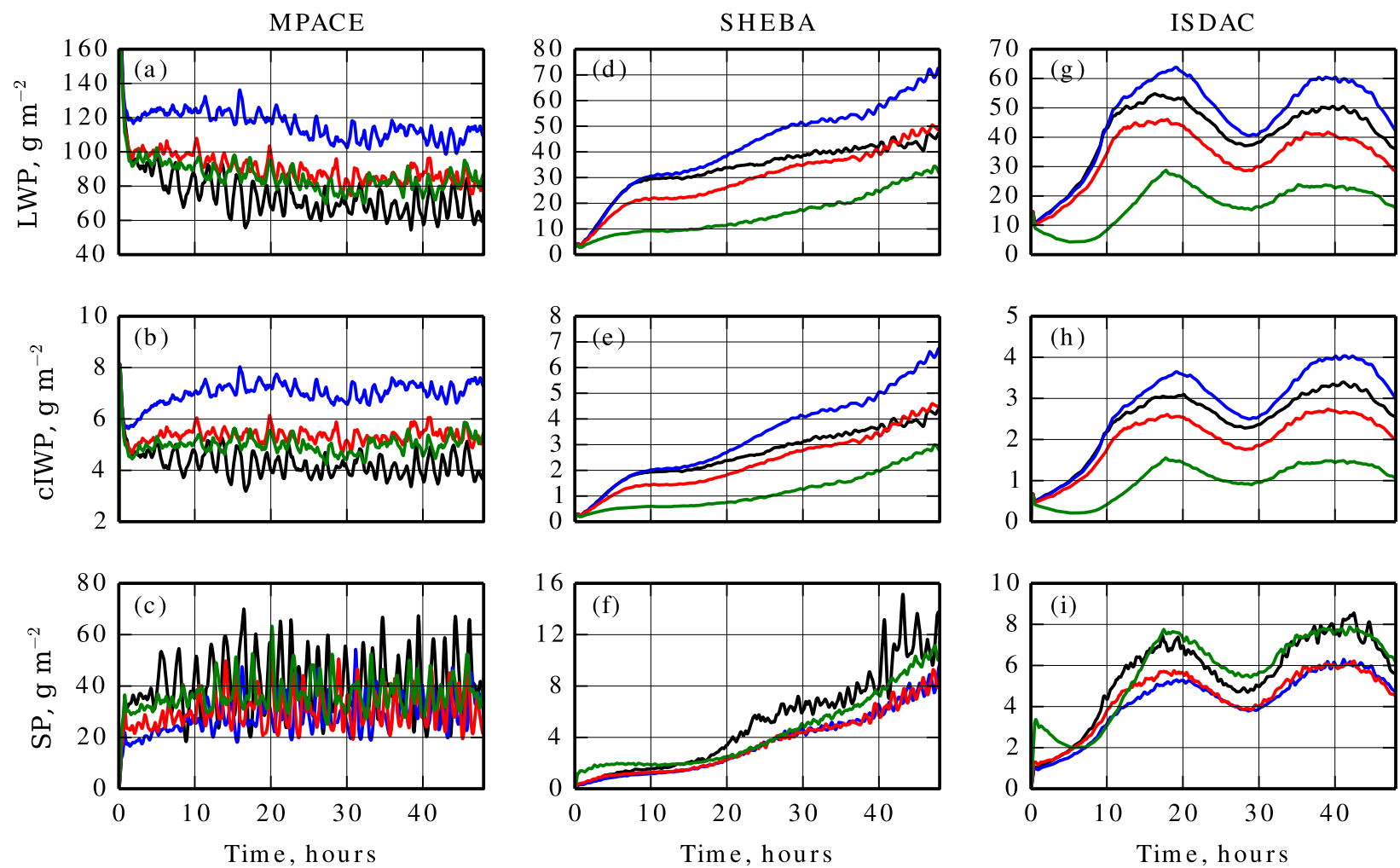

FIG. 11. (top) Liquid water path (LWP), (middle) cloud ice water path (cIWP), and (bottom) snow path (SP) sensitivity to the parameter $N_{0, s}$ with diagnosed values using M11 expression (black) and SS70 expression (green) and fixed values of $1 \times 10^{6} \mathrm{~m}^{-4}$ (lo_fix; blue) and $1 \times 10^{7} \mathrm{~m}^{-4}$ (hi_fix; red): (a)-(c) MPACE, (d)-(f) SHEBA, and (g)-(i) ISDAC. All use $n=0.1$ and $N_{0, i}=1 \times 10^{7} \mathrm{~m}^{-4}$.

the lower moist stable layer into the cloud-containing layer above.

Some additional results from our baseline simulations are shown in appendix $\mathrm{C}$, with comparisons to observational data or intercomparison results provided as possible.

\section{c. Sensitivity to $N_{0, s}$}

Simulation results show sensitivity to how the snow PSDF intercept parameter, $N_{0, s}$, is determined. Four options are considered in the results shown in Fig. 11. The first option, referred to as M11, is the diagnostic relationship given by Morrison et al. (2011a), which was used to carry out the baseline simulations presented in the preceding sections. The second option, which will be referred to as SS70, is the diagnostic relationship developed by Sekhon and Srivastava (1970), implemented similarly to the work of Reisner et al. (1998) but using the snow terminal velocity expression of Grabowski (1998), which is used elsewhere in our scheme. Both these expressions for $N_{0, s}$ are listed in appendix A. The remaining two options are fixed values of $N_{0, s}$ set at $1 \times 10^{6}$ and $1 \times 10^{7} \mathrm{~m}^{-4}$, where the latter of these is a commonly assumed value (e.g., Grabowski 1998). These will be referred to as the "lo_fix" and "hi_fix" cases, respectively.

Values of $N_{0, s}$ predicted by the SS70 and M11 expressions are plotted in Fig. 12. The M11 relationship predicts values of $N_{0, s}$ that are low at the top of the cloud but rapidly increase through the depth of the cloud to reach a broad maximum in the vicinity of the cloud base. The SS70 relationship predicts peak values of $N_{0, s}$ close to the cloud top and minimum values near the cloud base. Except for the upper part of the cloud, the M11 expression predicts $N_{0, s}$ values in MPACE that are roughly an order of magnitude higher than the SS70 expression. The opposite holds for SHEBA and ISDAC, in which the SS70 expression predicts higher $N_{0, s}$ from cloud top to the surface.

The various expressions for $N_{0, s}$ [combined with our assumed mass-diameter relationship; Eq. (A3)] correspond to different scalings between the snow mixing ratio $q_{s}$, snow particle concentration $n_{s}$, and average particle mass $m_{s}$. If a fixed value of $N_{0, s}$ is used, $n_{s} \propto q_{s}^{1 / 3}$ and $m_{s} \propto q_{s}^{2 / 3}$. A dependence on $N_{0, s}$ is also retained, $n_{s} \propto N_{0, s}^{2 / 3}$ and $m_{s} \propto N_{0, s}^{-2 / 3}$. The M11 expression yields the proportionalities of $n_{s} \propto q_{s}^{0.777}$ and $m_{s} \propto q_{s}^{0.223}$, while the SS70 relationship results in $n_{s} \propto q_{s}^{-0.403}$ and $m_{s} \propto q_{s}^{1.403}$. 

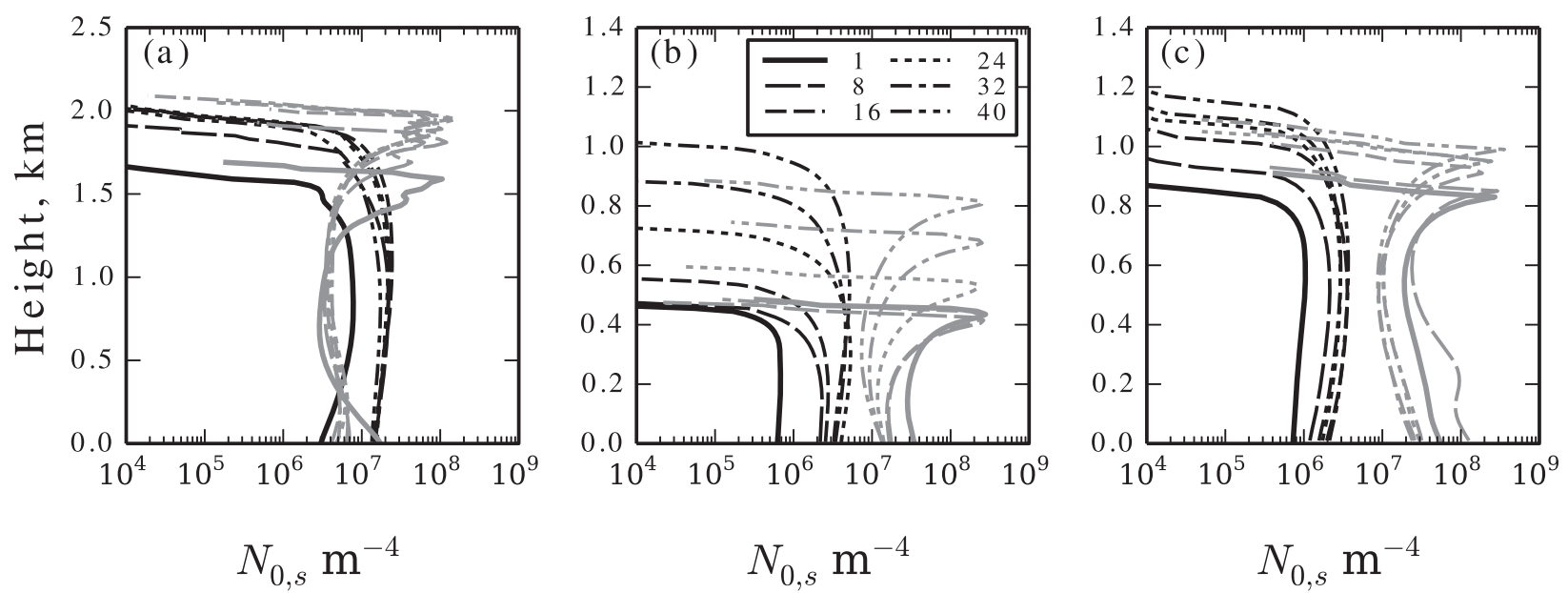

FIG. 12. Estimates of $N_{0, s}$ using the M11 (black) and SS70 (gray) expressions averaged over the hour-long windows indicated in the panel (b) legend. Results are shown for (a) MPACE, (b) SHEBA, and (c) ISDAC.

This means that the M11 expression predicts that the concentration of snow particles increases as the total mass of snow increases, while the SS70 expression predicts the inverse-namely, that the snow particle concentration decreases as the total mass of snow increases. Both expressions predict that the average particle mass increases as the total mass of snow increases, but the rate of increase is much higher using the SS70 relationship. The M11 expression can be associated with a physical scenario in which the mass of snow is increased mainly by the addition of more snow particles with limited particle growth. In contrast, the SS70 expression is suggestive of a scenario in which snow particles participate in aggregation processes, undergoing both self-collection and collection of cloud condensate to increase the mass of snow while decreasing the number concentration. In the fixed $N_{0, s}$ approach, $n_{s}$ increases with $q_{s}$, but does so more weakly than for the M11 expression, and the change in average particle mass is intermediate between the two diagnostic relationships.

We can also consider the implications of these options for setting $N_{0, s}$ on the behavior of the deposition/sublimation [Eq. (A19)] and aggregation source terms for snow [Eqs. (A10) and (A11)]. The deposition/sublimation term scales like $q_{s}^{0.924}$ using the M11 expression and like $q_{s}^{0.299}$ using the SS70 expression. For fixed $N_{0, s}$, the deposition/sublimation term varies as $N_{0, s}^{1 / 3} q_{s}^{2 / 3}$. The deposition/sublimation term is also linearly dependent on the supersaturation with respect to ice. The aggregation term (neglecting aggregation between snow and rain, which is unimportant in all these simulations) scales like $q_{s}^{1.108}$ using the M11 expression and like $q_{s}^{1.177}$ using the SS70 expression. For fixed $N_{0, s}$, the aggregation term varies as $N_{0, s}^{-0.083} q_{s}^{1.083}$. The aggregation term is also linearly dependent on the mixing ratio of cloud condensate, $q_{l}+q_{i}$. Thus, the deposition term is directly more sensitive to which method is used to set $N_{0, s}$, but enhancing deposition increases $q_{s}$ and can indirectly enhance the aggregation rate, depending on how the amount of cloud condensate changes.

Finally, the snow precipitation flux also scales with $q_{s}$ : as $q_{s}^{1.037}$ for the M11 expression, as $q_{s}^{1.176}$ for the SS70 expression, and as $N_{0, s}^{-1 / 12} q_{s}^{13 / 12}$ with fixed $N_{0, s}$. Recall, however, that the sedimentation tendency depends on the divergence of the precipitation flux and therefore the vertical structure of $q_{s}$ matters as well as the local value.

These relationships between snow mixing ratio and snow tendencies are most easily applied in the first few hours of the simulations, since all runs begin with the same initial conditions. Before proceeding further with this analysis, it should be noted that the usual initialization procedure for large-eddy simulations has some shortcomings. Simulations are initialized with random fluctuations of a given amplitude added to the mean values of moisture and temperature within the boundary layer. These fluctuations are generated without spatial correlation and without any correlation between variables and therefore are significantly different from realistic patterns of variability in these fields. Their purpose is merely to produce some initial buoyancy fluctuations that can generate vertical motion. However, this also means that the initial velocity fields do not exhibit well-developed turbulence. Feedbacks between turbulence and cloud properties are likely impacted. Nudging profiles through the depth of the boundary layer and/or turning off ice microphysical processes during a spinup period are possible, albeit still artificial, 
means of reducing the impact of the initial fluctuations. A theoretically more appealing approach is to generate initial fields that have more realistic structure, but developing such an approach is outside the scope of this study. A second artifact of simulation startup is apparent when we consider column-integrated quantities. Since the initial condition is snow free, finite time is needed for newly formed snow to fall below the cloud, where it may undergo sublimation, and eventually sediment through the depth of the boundary layer and be lost to the surface. For this reason, the column-integrated transport tendency is zero for a period of about $1.5-2.5 \mathrm{~h}$, and the MPACE and ISDAC cases show an early peak in depositional growth before competing sublimation losses occur (see Figs. 3c, 6c, and 9c).

Returning to Fig. 11, SHEBA and ISDAC simulations show similar responses to the four options. The highest amounts of cloud condensate occur in the lo_fix runs, followed by the M11 and hi_fix runs. The lowest cloud liquid and ice water paths are obtained in the SS70 runs. Over the first several hours, the SS70 simulations give the highest snow paths, corresponding to the lowest amounts of cloud condensate. This difference in the amount of snow is driven by the large depositional sources of the SS70 runs. These are several times larger than in the other runs, which is to be expected given the scaling relationships between $q_{s}$ and deposition rate presented above and the fact that autoconversion rates are nearly identical among all runs. Subsequently, this correlation between cloud condensate and snow amounts does not necessarily hold, as the snow paths of the M11 runs increase to be nearly as high as, or higher than, the SS70 runs' snow paths. This occurs because the autoconversion rates in the SS70 runs decrease relative to the other simulations. Sedimentation tendencies also adjust, so that the total tendency in snow amount is small.

Admittedly, snow path does not necessarily reflect surface precipitation fluxes. Therefore, accumulated surface snowfall was also checked for these simulations (not shown) and was also found to not be directly relatable to the differences in cloud condensate amounts. The differences in cloud liquid and ice water paths are not explained simply by the depletion of moisture through precipitation fluxes. Other effects, such as changes in cloud-top radiative cooling, come into play. Since both the SHEBA and ISDAC cases have zero or near-zero surface fluxes of latent and sensible heat, radiative cooling is crucial for driving turbulence and the effect of reducing the cloud-top cooling rate can be seen in profiles of the vertical velocity variance and turbulent kinetic energy (not shown). This weakens the updrafts that lift moisture into the cloud and enhance condensation.
Among the MPACE simulations, the lo_fix run again produces the greatest amount of cloud condensate, followed by the hi_fix and SS70 runs, which predict similar cloud liquid and ice water paths. The M11 run has the lowest cloud liquid and ice water paths. However, in all runs, the clouds remain quite optically thick and undergo high rates of cloud-top radiative cooling. Additionally, large surface heat fluxes contribute to the production of turbulence. Therefore, all runs maintain strong turbulence compared to SHEBA and ISDAC simulations. Cloud liquid and cloud ice water paths have an inverse relationship to snow path and accumulated surface precipitation.

A striking feature of the MPACE simulations are the fluctuations in cloud liquid, cloud ice, and (especially) snow path. These fluctuations are largest using the M11 expression for $N_{0, s}$. Fluctuations in snow path are also notable in the SHEBA and ISDAC simulations that use the M11 expression, but their amplitude is smaller because $q_{s}$ values are smaller. The relationship between $q_{s}$ and the deposition/sublimation rate implicit in the use of the M11 expression tends to increase the magnitude of fluctuations in each of these process rates considered separately, even if these fluctuations are partially cancelled out considering a net rate of deposition. The initialization procedure for the simulation, as described above, produces temperature and moisture fields that vary on small scales. As the simulation proceeds, the dominant scales of variability become larger and larger owing to the interaction between deposition/sublimation, buoyancy, and vertical motion. Eventually, the simulation domain contains a single area of large positive fluctuations in $q_{l}$ and $q_{i}$, which promote the formation of snow. This snow sediments and sublimates below the cloud base, producing a patch of negative buoyancy. In turn, this inhibits moisture transport back into the cloud, and $q_{l}, q_{i}$, and $q_{s}$ decrease before the cycle repeats at another location. Using a larger domain reduces the fluctuations in bulk statistics such as liquid water path because a few structures can be accommodated simultaneously, but growth in the size of the structures still occurs. Furthermore, it was confirmed that deposition/sublimation is the main driver of this phenomenon rather than (for example) variations in radiative cooling rates.

The fluctuating behavior of the simulations that use the M11 expression suggest that this formulation may insufficiently account for the importance of depositional growth of snow particles in these simulations. As mentioned above, the M11 expression is associated with a strong increase in snow concentration, but a weak increase in particle mass, as snow mixing ratio increases. This mismatch is especially true in the MPACE case, in which more snow mass is added by depositional growth 

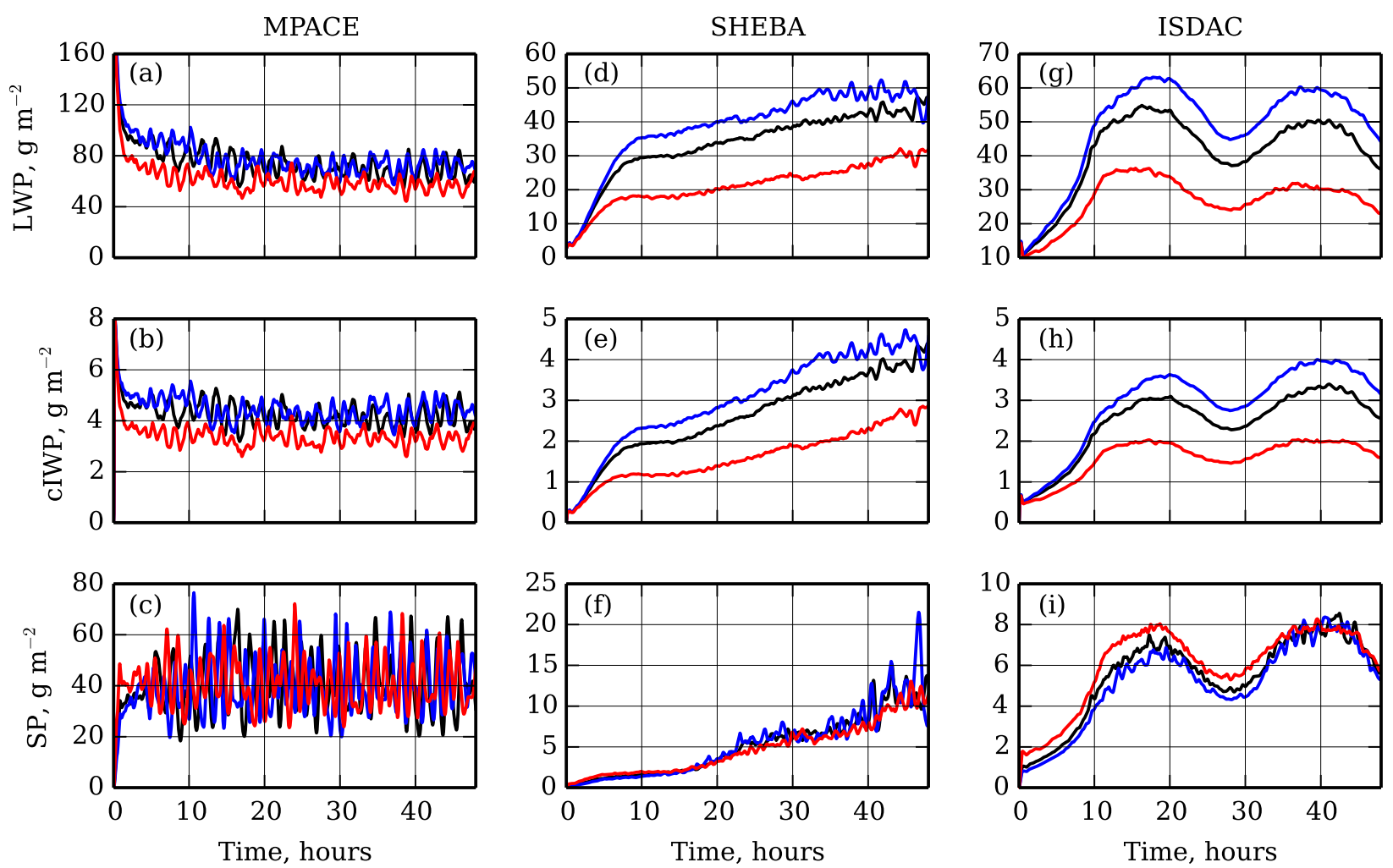

FIG. 13. (top) Liquid water path (LWP), (middle) cloud ice water path (cIWP), and (bottom) snow path (SP) sensitivity to the parameter $N_{0, i}$ with values of $5 \times 10^{6} \mathrm{~m}^{-4}$ (blue), $1 \times 10^{7} \mathrm{~m}^{-4}$ (black), and $5 \times 10^{7} \mathrm{~m}^{-4}$ (red): (a)-(c) MPACE, (d)-(f) SHEBA, and (g)-(i) ISDAC. All use $n=0.1$.

of existing snow particles than by addition of new snow particles through autoconversion (see Fig. 3). Nonetheless, the M11 expression remains more plausible a priori than the SS70 expression, whose prediction that snow number concentration decreases as $q_{s}$ increases seems tenuous given the typical balance of microphysical processes acting in AMPS. The M11 expression also has the advantage of being based on observations of Arctic clouds, rather than on clouds in other regions. Additionally, instantaneous fluctuations in bulk statistical quantities such as LWP are greatly reduced when they are computed as averages over larger simulation domains, so the real question is what constitutes realistic frequencies and amplitudes of local variability. Spectral analyses of observed clouds like that of Shupe et al. (2008) are important in this regard.

\section{d. Sensitivity to $N_{0, i}$}

Figure 13 compares simulation results as the ice PSDF intercept parameter $N_{0, i}$ is varied from $5 \times 10^{6}$ to $5 \times 10^{7} \mathrm{~m}^{-4}$. Assuming that cloud ice particles have a spherical geometry results in mean particle size changing as $N_{0, i}^{-3 / 4} q_{i}^{3 / 4}$, while cloud ice particle concentrations $n_{i}$ vary as $N_{0, i}^{3 / 4} q_{i}^{1 / 4}$. Therefore, an order of magnitude change in $N_{0, i}$ corresponds to more than a fivefold variation in ice particle concentration if $q_{i}$ is held constant. In actuality, we find that the values of the cloud ice mixing ratio decrease in our simulations when $N_{0, i}$ is increased. However, because $n_{i}$ varies weakly with $q_{i}$, the change in simulated ice particle concentrations remains dominated by the change in $N_{0, i}$. Increasing $N_{0, i}$ consistently leads to decreases in cloud ice and liquid water paths as a result of depletion of moisture from the cloud top through enhanced autoconversion and sedimentation of snow. Integrated surface precipitation amounts also increase with $N_{0, i}$ and snow path is either weakly affected or increased.

The variation of the snow autoconversion term [Eq. (A7)] with changes in $N_{0, i}$ and $q_{i}$ is plotted in Fig. 14a. All other conditions, including the humidity and temperature of the environment, are assumed to be held constant. Except for combinations of a very small cloud ice mixing ratio and high value of $N_{0, i}$, the autoconversion source term increases for fixed $q_{i}$ as $N_{0, i}$ is increased. With $q_{i}=1 \times 10^{-5} \mathrm{~kg} \mathrm{~kg}^{-1}$ (taken as a typical value for the clouds simulated here), the autoconversion source term increases linearly with $N_{0, i}$ : the autoconversion source changes by a factor of 10 as $N_{0, i}$ is 
(a)

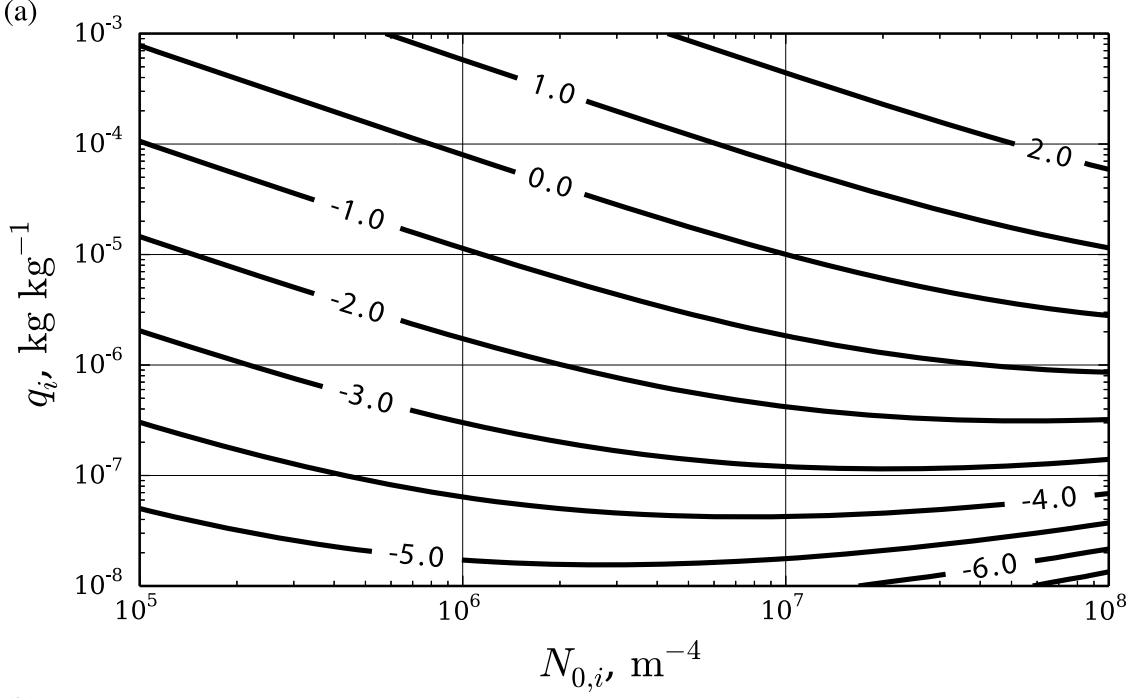

(b)

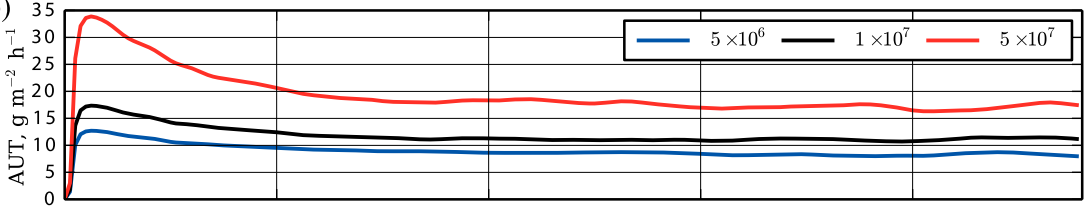

(c)

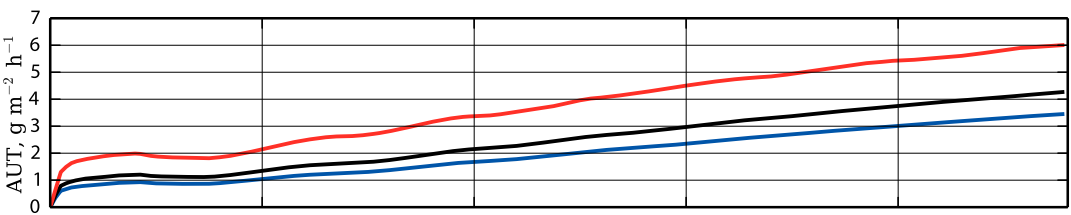

(d)

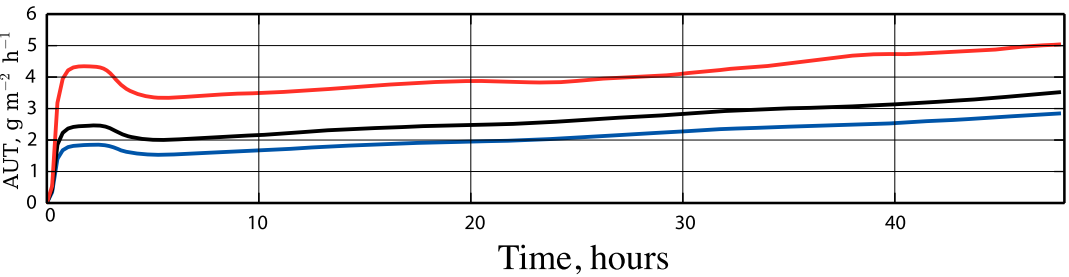

FIG. 14. (a) Variation of the snow autoconversion source term [Eq. (A7)] with changes in cloud ice mixing ratio $q_{i}$ and ice PSDF intercept parameter $N_{0, i}$. Contours are plotted as $\log _{10}\left[\operatorname{AUT}\left(q_{i}, N_{0, i}\right) / \operatorname{AUT}\left(q_{i}=1 \times 10^{-5}, N_{0, i}=1 \times 10^{7}\right)\right]$ with $q_{i}$ in $\mathrm{kg} \mathrm{kg}^{-1}$ and $N_{0, i}$ in m${ }^{-4}$. (b)-(d) Snow autoconversion source term integrated over boundary layer depth in $\mathrm{g} \mathrm{m}^{-2} \mathrm{~h}^{-1}$ for MPACE, SHEBA, and ISDAC, respectively. Line color indicates value of $N_{0, i}$ in $\mathrm{m}^{-4}: 5 \times 10^{6}$ (blue), $1 \times 10^{7}$ (black), and $5 \times 10^{7}$ (red).

increased from $10^{6}$ to $10^{7} \mathrm{~m}^{-4}$ and increases by another factor of 10 taking $N_{0, i}$ from $10^{7}$ to $10^{8} \mathrm{~m}^{-4}$. If instead $N_{0, i}$ is held fixed and $q_{i}$ is decreased by an order of magnitude, the autoconversion source term decreases by slightly more than an order of magnitude.

Figures 14b-d show how the column-integrated snow autoconversion source terms with changing $N_{0, i}$ in the MPACE, SHEBA, and ISDAC cases. In all cases, the tenfold change in $N_{0, i}$ produces much less than a tenfold change in the autoconversion source; instead, the integrated source term actually changes by a factor of 2-3.
The change in $N_{0, i}$ is mitigated by two factors. First, peak values of $q_{i}$ decrease with increased $N_{0, i}$. Second, the cloud becomes thinner as $N_{0, i}$ increases and autoconversion takes place over a smaller depth. Both these factors are consistent with the reduced cloud ice water paths seen in Fig. 13.

Whether the increased autoconversion rate increases the snow path is case dependent. The relationship between changing $N_{0, i}$ and snow path appears to depend on the relative importance of autoconversion and deposition/sublimation source terms and the shape of the 
thermodynamic profiles. Since the autoconversion term parameterizes the growth of ice crystals through vapor deposition, in some sense ice and snow particles compete for the available water vapor in excess of the saturation vapor pressure with respect to ice when they are collocated. Furthermore, there is not a one-to-one tradeoff between depositional growth rates of ice and of snow, even at the same location, because of the different assumed particle shapes, capacitances, and ventilation effects [see Eqs. (A7) and (A19) in appendix A]. However, in some cases, the regions of peak autoconversion and deposition are more separated vertically and there is less competition between the processes. For example, in ISDAC, increasing $N_{0, i}$ strongly increases autoconversion near cloud top without inhibiting deposition lower in the cloud. In SHEBA, regions where deposition and autoconversion occur mostly overlap (see Fig. 6) and snow path is increased by increasing $N_{0, i}$ only early in the simulation while the boundary layer is moistening. In the MPACE case, snow path is initially higher for the $N_{0, i}=5 \times 10^{7} \mathrm{~m}^{-4}$ run, but all three runs recover the same average snow path $\left(41 \mathrm{~g} \mathrm{~m}^{-2}\right)$ over their final $24 \mathrm{~h}$, as the increase in autoconversion rates associated with increasing $N_{0, i}$ is balanced by declines in growth due to accretion and deposition.

\section{e. Sensitivity to $n$}

The parameter $n$ determines the mixed-phase saturation vapor pressure and the liquid fraction of cloud condensate at a given temperature (see section 2a). Because of this dual role, the simulated clouds show relatively more complex responses to changes in $n$ than to changes in $N_{0, i}$. Variations in cloud liquid, cloud ice, and snow amounts with changing $n$ are plotted in Fig. 15. Sensitivities to $n$ depend on the thermodynamic characteristics of the boundary layer, since the MPACE case responds differently than the ISDAC and SHEBA cases, which are colder and/or drier. In SHEBA and ISDAC, increasing $n$ increases cloud IWP at the expense of LWP. However, the tradeoff between liquid and ice is not one to one and the total amount of cloud condensate decreases. In MPACE, LWP holds nearly constant as $n$ is increased from 0.05 to 0.1 but increases by about $10 \%$ as $n$ is increased to 0.5 . Cloud ice water path increases with increasing $n$, with a large jump occurring as $n$ is varied from 0.1 to 0.5 . Consequently, the total amount of cloud condensate increases with increasing $n$ in the MPACE results.

To try to explain these trends, Fig. 16 decomposes how several quantities vary with temperature and $n$. The range of temperatures shown is restricted to values relevant for the clouds simulated here. Calculations are performed assuming $q_{t}=1.5 \mathrm{~g} \mathrm{~kg}^{-1}$, which is the value of $q_{t}$ through the mixed layer in the initial moisture profile of the ISDAC case. Decreasing $q_{t}$ shifts the contours to lower temperatures while the shape of the contours remains similar; the converse is true for increasing $q_{t}$. Therefore, this analysis is simplified relative to the results shown in Fig. 15 because $q_{t}$ is held constant. Nevertheless, it helps to illustrate the tradeoffs that occur as $n$ is varied.

Figure 16a plots the cloud liquid mixing ratio $q_{l}$. At a given temperature, $q_{l}$ is nearly constant as $n$ is increased from 0.01 to 0.1 . The decrease in liquid fraction is compensated by the increase in total cloud condensate because of the lowering of the saturation vapor mixing ratio. At higher values of $n$, with the exact value dependent on the temperature, $q_{l}$ begins to decrease precipitously as the depression of the saturation vapor mixing ratio no longer compensates the lowering of the liquid fraction. The cloud ice mixing ratio (Fig. 16b) shows different behavior, as $q_{i}$ increases rapidly with increasing $n$ at a given temperature as a result of the combination of increasing ice fraction and decreasing saturation vapor mixing ratio. Figure 16c shows the supersaturation with respect to ice-that is, the quantity denoted by $S_{i}-1$ in appendix A, expressed as a percent. At temperatures where saturation occurs, the trends in supersaturation are similar to the trends in $q_{l}$. In subsaturated conditions (here, for $T>262 \mathrm{~K}$ ), all dependence on $n$ is lost, and $S_{i}-1$ depends only on temperature and $q_{t}$. Finally, Fig. 16d plots the snow autoconversion source term [Eq. (A7)] assuming $N_{0, i}=1 \times 10^{7} \mathrm{~m}^{-4}$. In contrast to Fig. 14a discussed in the preceding section, Fig. 16d combines the effects of changing $q_{i}$, temperature, and supersaturation. The contours reflect this interaction of several effects: at lower values of $n$, the autoconversion rate increases as $q_{i}$ increases, while autoconversion rates drop as a result of falling supersaturation at higher values of $n$. Quantitatively, the demarcation between "low" and "high" $n$ depends on temperature. In the cases we simulate, we fall in the regime where the autoconversion rate increases with $n$.

Also shown in Fig. 16 are the column-integrated snow autoconversion (Fig. 16e) and deposition-sublimation (Fig. 16f) source terms of the ISDAC simulations. Consistent with the results shown in Fig. 16d, the autoconversion source term increases as $n$ is raised from 0.05 to 0.5 , and this sensitivity is maintained for the duration of the simulations. The higher autoconversion source increases $q_{s}$, in turn boosting the deposition rate over the first few hours (Fig. 16f), following the analysis in section 4c. The higher values of $q_{s}$ outweigh lower ice supersaturation during this initial period. After a few hours, the sensitivity of the deposition/sublimation rate to $n$ 

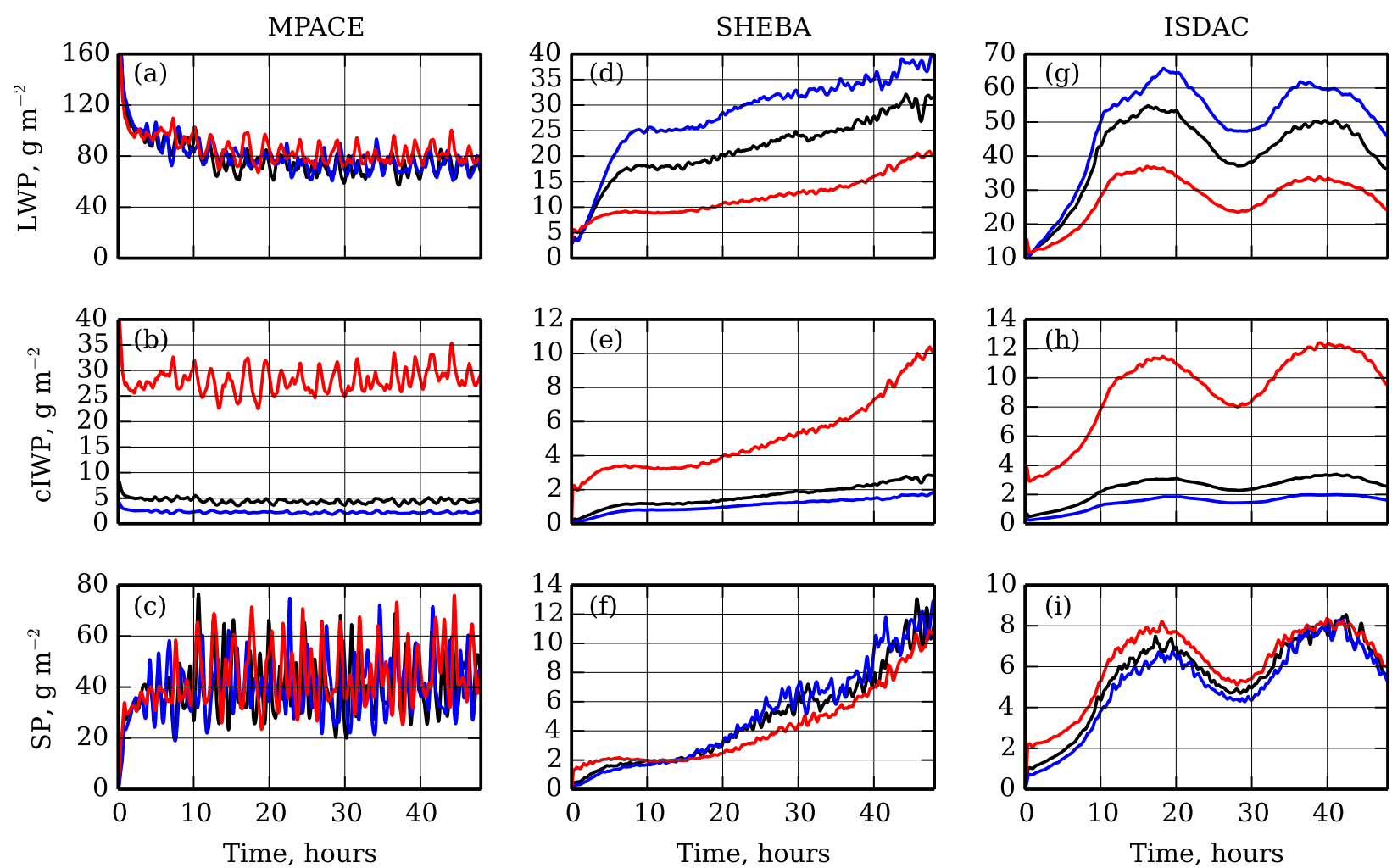

FIG. 15. (top) Liquid water path (LWP), (middle) cloud ice water path (cIWP), and (bottom) snow path (SP) sensitivity to the parameter $n$ with values of 0.05 (blue), 0.1 (black), and 0.5 (red). (a)-(c) MPACE results with $N_{0, i}=5 \times 10^{6} \mathrm{~m}^{-4}$, (d)-(f) SHEBA results with $N_{0, i}=5 \times 10^{7} \mathrm{~m}^{-4}$, and (g)-(i) ISDAC results with $N_{0, i}=1 \times 10^{7} \mathrm{~m}^{-4}$.

reverses. By hour 20, the simulation using $n=0.5$ has a net sublimation loss of snow while simulations with $n=0.05$ and 0.1 maintain a small net depositional gain. This occurs because the cloud is shallower and ice supersaturation values within the cloud are lower with higher $n$, thus reducing the deposition rate. However, the value of $n$ has no direct effect on values of subsaturation, and sublimation rates below the cloud are controlled by the value of $q_{s}$. Therefore, sublimation rates increase with $n$.

All cases show an increase in autoconversion rates as $n$ is increased, but depositional growth rates decrease (considering either deposition alone or the combined effects of deposition and sublimation). Similar to the $N_{0, i}$ sensitivity tests, the comparative magnitudes and locations of autoconversion and deposition determine the response to changes in $n$. In MPACE, the enhancement of vapor deposition as $n$ is reduced is more significant than the suppression of autoconversion, so total cloud condensate decreases with decreasing $n$. In SHEBA and ISDAC, the enhancement of autoconversion rates with higher $n$ at first dominates the decline in deposition, leading to higher snowfall rates and lower amounts of cloud condensate. As the clouds evolve, the balance between autoconversion and deposition shifts and the runs with higher values of $n$ begin to produce less snow. However, cloud condensate amounts continue to show the effects of the earlier losses.

Therefore, the trends in cloud liquid and ice water path shown in Fig. 15 can be explained by a combination of the direct effects of changing $n$, as illustrated in Figs. 16a-d, and the indirect effects associated with changes in the boundary layer structure, which in turn can be related to different rates of snow production and precipitation.

\section{Conclusions}

The results of a series of model intercomparison studies based on observations from the MPACE (Klein et al. 2009), SHEBA/FIRE-ACE (Morrison et al. 2011b), and ISDAC (Ovchinnikov et al. 2014) campaigns have strikingly demonstrated the challenges in simulating Arctic mixed-phase stratocumulus clouds (AMPS). Since ice initiation mechanisms in the atmosphere are not well understood (Cantrell and Heymsfield 2005), these difficulties are not surprising. However, even when important aspects of the ice microphysical scheme are constrained or 

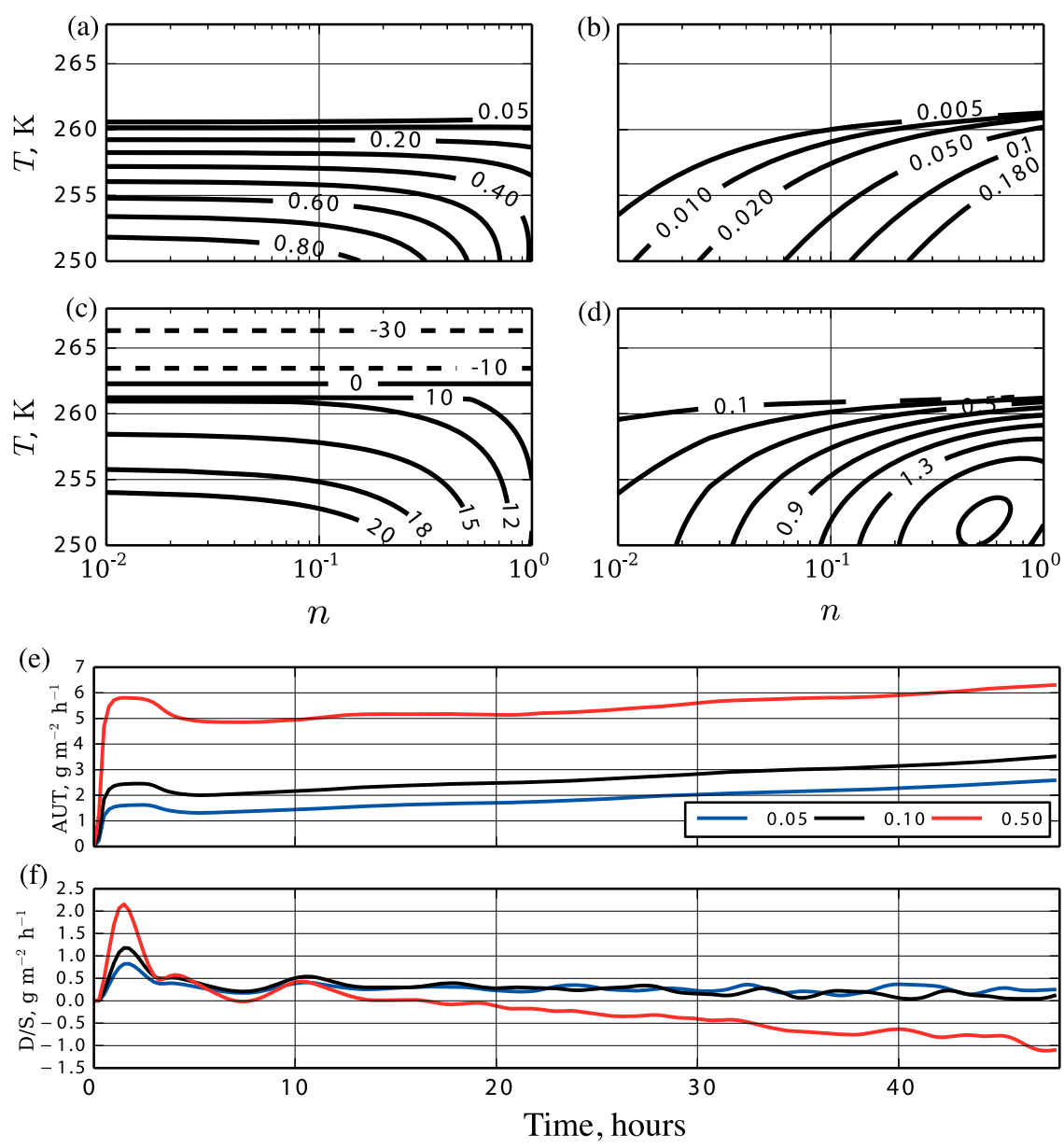

FIG. 16. Variations with $n$ of (a) $q_{l}$ and (b) $q_{i}$ in $\mathrm{g} \mathrm{kg}^{-1}$. (c) Supersaturation with respect to ice in percent. (d) Snow autoconversion source term in $\mathrm{g} \mathrm{kg}^{-1} \mathrm{day}^{-1}$. All values are computed assuming $q_{t}=1.5 \mathrm{~g} \mathrm{~kg}^{-1}$, and the autoconversion calculation uses $N_{0, i}=1 \times 10^{7} \mathrm{~m}^{-4}$. (e),(f) Snow source term integrated over boundary layer depth in $\mathrm{g} \mathrm{m}^{-2} \mathrm{~h}^{-1}$ for the ISDAC case for $n=0.05$ (blue), 0.10 (black), and 0.50 (red) for autoconversion and deposition-sublimation, respectively.

standardized, model results vary (Morrison et al. 2011b; Ovchinnikov et al. 2014). These findings highlight the sensitivity of simulations of mixed-phase clouds to their microphysical parameterizations. However, it should be noted that the results of liquid-only runs in the MPACE and ISDAC intercomparisons differed significantly among the various models, indicating that differences in model dynamics also play a role. This is consistent with intercomparisons of LES of lower-latitude, liquid-phase stratocumuli, which also find noteworthy discrepancies among participating models (e.g., Stevens et al. 2005; Ackerman et al. 2009). Thus, it is important to understand how turbulence closures, numerical schemes, and other factors combine with microphysical parameterizations to determine simulation results. Properly characterizing cloud-scale circulations is important because the maintenance of AMPS may depend on a balance between the time scales of vertical motion and of competing microphysical processes, as in the conceptual model advanced by Shupe et al. (2008). Moreover, Arctic stratocumuli exist under a broad range of conditions, with wide variations in the strengths of surface fluxes, shear, and large-scale forcings. Therefore, a variety of different feedbacks between turbulence and microphysics may be important depending on the circumstances.

Generally speaking, past LES studies of AMPS have tended to prioritize microphysical modeling issues. With increasing computational power comes a trend toward developing ever more sophisticated schemes to handle the complexities of mixed-phase clouds. Nonetheless, we argue that there is a continued role for simpler 
microphysical schemes as part of systematic approaches to bridging simulations across different scales and building understanding of how different model components interact. Here, we develop a simple microphysical scheme with the goal of capturing important features of AMPS that strongly interact with the generation of turbulence. It neglects the details of ice initiation, which remain difficult for even more sophisticated models to predict owing to theoretical uncertainties and insufficient observational constraints, and uses a simple representation of ice crystal shape that is most realistic only for very small ice crystals. A diagnostic approach is used to determine the amount and phase of cloud condensate. While this diagnostic approach greatly simplifies the scheme, it precludes an explicit representation of the growth processes of cloud droplets and ice particles. Furthermore, cloud condensate is assumed to move with the gas phase; sedimentation of cloud ice and liquid droplets is neglected. Therefore, this model is not intended to provide a totally realistic representation of all the phenomena important to AMPS. Rather, it is meant to serve as a tool whose characteristics can be well understood precisely because of its simple formulation. The new, simplified scheme is used to simulate the MPACE, SHEBA, and ISDAC cases based on the setups used in their respective intercomparison studies. Using a baseline set of parameters for the microphysical scheme with no case-specific tuning, statistics of liquid and ice water path are found to be in reasonable agreement with the results presented in the intercomparisons.

Simulation results are assessed for their sensitivity to three key parameters of the microphysical scheme: the intercept parameter of the snow particle distribution $N_{0, s} ;$ the analogous quantity for the ice particle distribution $N_{0, i}$; and the exponent $n$ appearing in the temperature-dependent phase-partition function, which is used to diagnose mixing ratios of cloud liquid and cloud ice. Along with specifying fixed values of $N_{0, s}$, two different empirical expressions for $N_{0, s}$ were also tested: an older expression based on the work of Sekhon and Srivastava (1970) and a newer expression developed in Morrison et al. (2011a) using observations of Arctic clouds. The latter expression was found to reasonably predict time-averaged statistics, while tending to increase the variability of hydrometeor amounts on hourly time scales due to interactions between turbulent motions and sublimation of snow. All cases show some dependence on each of the three parameters. In terms of bulk statistics, cloud liquid and ice water path show the most sensitivity while snow path is weakly affected for most of the simulation duration. However, snow path sensitivity can be much more pronounced over the first several hours, apparently giving rise to lasting effects. First, as a direct effect, the cloud layer is depleted of moisture. As a consequence of the diminished liquid water content, cloud-top radiative cooling is diminished and turbulence levels decrease. This latter effect is particularly important in the SHEBA and ISDAC cases, which have weak or zero surface fluxes. The MPACE case responds somewhat differently to variations in parameter values because it features stronger surface fluxes, a higher cloud-top temperature, and heavier precipitation with a greater contribution of aggregation processes to the growth of snow.

The sensitivity of bulk statistics such as liquid water path reflects the sensitivity of microphysical processes to parameter values, as modulated by interactions between microphysical processes and their feedbacks to dynamics. The importance of these modulations can already be seen in the fact that a mixed-phase cloud is maintained in all the sensitivity test simulations for their entire length of $48 \mathrm{~h}$, even when initial precipitation rates are high. Increasing either the ice or snow intercept parameters, $N_{0, i}$ or $N_{0, s}$, decreases the liquid water path. However, this occurs through different pathways: increasing $N_{0, i}$ has the direct effect of increasing the autoconversion rate of ice into snow, while increasing $N_{0, s}$ has the direct effect of increasing the rate of snow growth through vapor deposition. Different processes predominate at different levels within the cloud and therefore can set up different feedbacks with dynamics. These feedbacks will be explored more completely in future work, along with analysis of the sensitivity of large-eddy simulations of AMPS to features such as numerical schemes and turbulence model formulations, which have received limited attention in the past.

Acknowledgments. This research was partly carried out at the Jet Propulsion Laboratory, California Institute of Technology, under a contract with the National Aeronautics and Space Administration.

\section{APPENDIX A}

\section{Description of Microphysical Processes}

The intercept parameter of the snow particle size distribution function (PSDF) can be estimated from an expression in the form

$$
N_{0, s}=x\left(\rho q_{s}\right)^{y}
$$

with values $x=4.36 \times 10^{9}, y=0.63$ from Morrison et al. (2011a) and $x=29.02, y=-1.105$ in the approach based on the work of Sekhon and Srivastava (1970) and 
Reisner et al. (1998). The units of $\rho q_{s}$ are $\mathrm{kg} \mathrm{m}^{-3}$. Similarly, $N_{0, r}$ is found from the relationship

$$
N_{0, r}=650.146\left(\rho q_{r}\right)^{-1.222}
$$

based on the work of Abel and Boutle (2012).

Particle mass and settling velocity are related to particle diameter by

$$
\begin{aligned}
& m_{x}=a_{x} D_{x}^{b_{x}} \text { and } \\
& v_{t, x}=c_{x} D_{x}^{d_{x}}
\end{aligned}
$$

with parameter values taken from Grabowski (1989) of $a_{r}=\rho_{l} \pi / 6, b_{r}=3, c_{r}=130, d_{r}=0.5$ for rain; $a_{s}=$ $2.5 \times 10^{-2}, b_{s}=2, c_{s}=4, d_{s}=0.25$ for snow; and $a_{i}=$ $\rho_{i} \pi / 6$ and $b_{i}=3$ for ice. Cloud ice and liquid droplets are currently assumed to have $v_{t}=0$, although nonzero sedimentation velocities are more realistic. Densities of liquid water, $\rho_{l}$, and ice, $\rho_{i}$, are respectively 1000 and $900 \mathrm{~kg} \mathrm{~m}^{-3}$. These parameter values for cloud ice correspond to dense, spherical particles. Such an assumed particle morphology is most consistent with small, unrimed crystals with maximum diameters of approximately 30-100 $\mu \mathrm{m}$ (e.g., Morrison and Grabowski 2008; Heymsfield et al. 2007), which our cloud ice category is intended to represent. However, these parameter values are uncertain because of the limited observations of small ice crystals and should be treated as potentially adjustable quantities within the scheme. An additional concern is the fact that the scheme lacks a mechanism to enforce the assumption of small ice particle size. This will be discussed further in the context of the snow autoconversion source term [Eq. (A7)].

The slope parameter of the PSDF is given by (e.g., Grabowski 1998, hereafter G98)

$$
\lambda_{x}=\left[\frac{a_{x} N_{0, x} \Gamma\left(b_{x}+1\right)}{\rho q_{x}}\right]^{1 /\left(b_{x}+1\right)},
$$

where $x$ denotes either rain $(r)$, snow $(s)$, or ice $(i)$. Morrison et al. (2011a) also provide an empirical expression for the slope parameter of the PSDF, $\lambda_{s}=$ $3.81 \times 10^{3}\left(\rho q_{s}\right)^{-0.147}$, that is used here along with their expression for $N_{0, s}$. In this case, the value of $a_{s}$ appears only in the expression for melting of snow in rain-snow collisions above $273.16 \mathrm{~K}$ [Eq. (A17)], which is never used in the simulations carried out in this work.

The mass-weighted fall speeds of rain and snow are then readily found to be given by

$$
V_{T, x}=c_{x} \frac{\Gamma\left(b_{x}+d_{x}+1\right)}{\Gamma\left(b_{x}+1\right)} \lambda_{x}^{-d_{x}} .
$$

Parameterization of the snow autoconversion rate follows Harrington et al. (1995), with the necessary adjustments to account for an exponential, rather than gamma, PSDF. With ice particle capacitance assumed to be equal to particle diameter and ventilation effects neglected, the source term is given by

$$
\begin{aligned}
& \left(\frac{\partial q_{s}}{\partial t}\right)_{\mathrm{AUT}} \\
& \quad=\frac{4 \pi}{\rho}\left(S_{i}-1\right) G_{i}(T) N_{0, i} \exp \left(-\lambda_{i} D_{b}\right)\left(\frac{D_{b}^{2}}{b_{i}}+\frac{1+\lambda_{i} D_{b}}{\lambda_{i}^{2}}\right) .
\end{aligned}
$$

In this expression, $S_{i}=r_{\mathrm{vs}, i} / r_{v}$ is the saturation ratio with respect to ice and $G_{i}$ is a thermodynamic quantity that can be approximated as (Pruppacher and Klett 1978; G98)

$$
G_{i}(T)=1 \times 10^{-7}\left[2.2 \frac{T}{e_{s, i}(T)}+\frac{220}{T}\right]^{-1} .
$$

Note that analogous quantities $S_{l}$ and $G_{l}$ are defined for saturation with respect to liquid. The threshold size $D_{b}$ is $125 \mu \mathrm{m}$. This threshold size scales the magnitude of the autoconversion source rather than acting as a strict bound on the size of cloud ice particles. Such a strict bound is inconsistent with the assumed exponential ice PSDF. Since a cutoff size on cloud ice particles cannot be imposed via the snow autoconversion term, the assumed typical small size of ice particles leading to the selected values of the parameters $a_{i}$ and $b_{i}$ cannot be enforced a priori in simulations. Instead, it would be necessary to adjust $N_{0, i}$ (and also possibly the phasepartition function exponent $n$ ) in a heuristic manner to obtain consistency, as changing $N_{0, i}$ modifies both the amount of cloud ice obtained in a simulation (by changing the snow autoconversion rate) and the size distribution of the cloud ice particles. However, $a_{i}$ and $b_{i}$ are only used to compute $\lambda_{i}$, which in turn is only used by Eq. (A7). Therefore, one possible view is that $a_{i}$ and $b_{i}$ are model parameters, like $D_{b}$ and $N_{0, i}$, which have a physical interpretation but whose practical role lies in adjusting the snow autoconversion rate.

The source term of $q_{r}$ due to autoconversion (AUT) follows the parameterization developed from the work of Berry and Reinhardt (1974), as expressed in SI units by Gilmore and Straka (2008). The number concentration of cloud droplets and their size dispersion are determined from the empirical formulas of Martin et al. (1994). Both this rain autoconversion parameterization and the snow autoconversion parameterization described above are free from any explicit threshold values of $q_{l}$ or $q_{i}$. 
However, for computational purposes a very low threshold value of $10^{-10} \mathrm{~kg} \mathrm{~kg}^{-1}$ is imposed to avoid numerical issues associated with division by small numbers.

A number of source terms arise from collection of one hydrometeor species by another. Following G98, these are referred to as accretion (ACC) terms. The scheme considers accretion sources and sinks due to raindroplet, rain-ice, rain-snow, snow-droplet, and snowice collisions. Graupel initiation is neglected because of the limited amount of riming that typically occurs in AMPS. The formulation of these source terms is largely based on Rutledge and Hobbs (1983, 1984) but using G98's sedimentation velocity expressions and collision and sticking efficiencies for ice and snow from Seifert and Beheng (2006). The efficiency of all collisions involving raindrops is assumed to be unity.

Following Grabowski (1989), the source term for rain mixing ratio due to collection of cloud droplets is

$$
\left(\frac{\partial q_{r}}{\partial t}\right)_{\mathrm{AGG} ., r-l}=\frac{\pi}{4} N_{0, r} c_{r} E_{\mathrm{rl}} q_{l} \frac{\Gamma\left(d_{r}+3\right)}{\lambda_{r}^{d_{r}+3}}
$$

and, similarly, the source term for snow mixing ratio due to collection of cloud ice is

$$
\left(\frac{\partial q_{s}}{\partial t}\right)_{\mathrm{AGG}, s-i}=\frac{\pi}{4} N_{0, s} \alpha_{s} c_{s} E_{\mathrm{si}} q_{i} \frac{\Gamma\left(d_{s}+3\right)}{\lambda_{s}^{d_{s}+3}},
$$

where $\alpha_{s}$ is a geometrical factor for snow with a value of 0.3 (G98).

Cloud droplets that are collected by snow are assumed to freeze for $T \leq 273.16 \mathrm{~K}$, providing a source term for snow. At higher temperatures, it is assumed the accreted droplets are shed as rain along with some additional melted snow as a result of enhanced melting (Rutledge and Hobbs 1984). Therefore, for $T \leq 273.16 \mathrm{~K}$, the source term for snow mixing ratio due to collection of liquid droplets is

$$
\left(\frac{\partial q_{s}}{\partial t}\right)_{\mathrm{AGG} ., s-l}=\frac{\pi}{4} N_{0, s} \alpha_{s} c_{s} E_{\mathrm{sl}} q_{l} \frac{\Gamma\left(d_{s}+3\right)}{\lambda_{s}^{d_{s}+3}} .
$$

Let the quantity given by Eq. (A11) be denoted as $\mathrm{RIME}_{\mathrm{sl}}$. For temperatures above $273.16 \mathrm{~K}$, the sink term for $q_{s}$ due to enhanced melting is

$\left(\frac{\partial q_{s}}{\partial t}\right)_{\mathrm{AGG} ., s-l}=-\frac{c_{w}}{L_{f}}(T-273.16) \mathrm{RIME}_{\mathrm{sl}}$

and the source term for $q_{r}$ due to enhanced melting and shedding of accreted cloud droplets is $\left(\frac{\partial q_{r}}{\partial t}\right)_{\mathrm{AGG} ., s-l}=\left[1+\frac{c_{w}}{L_{f}}(T-273.16)\right] \mathrm{RIME}_{\mathrm{sl}}$.

The thermodynamic parameters in these equations have the values $c_{w}=4218 \mathrm{~J} \mathrm{~kg}^{-1} \mathrm{~K}^{-1}$ and $L_{f}=3.34 \times 10^{5} \mathrm{~J} \mathrm{~kg}^{-1}$ (Rutledge and Hobbs 1984).

Collisions between rain and ice can be assumed to form snow, since our setting of $T_{\text {warm }}$ ensures that cloud ice does not exist at temperatures above $273 \mathrm{~K}$. The sink term for rain mixing ratio is given by

$$
\left(\frac{\partial q_{r}}{\partial t}\right)_{\mathrm{AGG} . r-i}=-\frac{\pi}{4} \frac{N_{0, i}}{\lambda_{i}} \frac{\Gamma\left(d_{r}+6\right)}{\lambda_{r}^{d_{r}+6}} N_{0, r} E_{\mathrm{ri}} a_{r} c_{r} \rho^{-1}
$$

while the source term for snow also accounts for the mass of the collected ice particles,

$$
\begin{aligned}
\left(\frac{\partial q_{s}}{\partial t}\right)_{\mathrm{AGG} . r-i}= & -\left(\frac{\partial q_{r}}{\partial t}\right)_{\mathrm{AGG} ., r-i} \\
& +\frac{\pi}{4} \frac{\Gamma\left(d_{r}+3\right)}{\lambda_{r}^{d_{r}+3}} N_{0, r} \alpha_{r} c_{r} E_{\mathrm{ri}} q_{i} .
\end{aligned}
$$

For further details, see the use of the terms PRACI and PIACR in Rutledge and Hobbs (1984), noting that we exclude the possibility of graupel initiation.

Collisions of rain and snow are assumed to form snow for temperatures below $273.16 \mathrm{~K}$. The source term for $q_{s}$ due to collection of rain is

$$
\begin{aligned}
\left(\frac{\partial q_{s}}{\partial t}\right)_{\mathrm{AGG} . r-s}= & \frac{\pi}{4}\left|V_{T, r}-V_{T, s}\right| N_{0, s} N_{0, r} a_{r} E_{\mathrm{sr}}\left[\frac{2 \Gamma\left(b_{r}+1\right)}{\lambda_{s}^{3} \lambda_{r}^{b_{r}+1}}\right. \\
& \left.+\frac{2 \Gamma\left(b_{r}+2\right)}{\lambda_{s}^{2} \lambda_{r}^{b_{r}+2}}+\frac{\Gamma\left(b_{r}+3\right)}{\lambda_{s} \lambda_{r}^{b_{r}+3}}\right] \rho^{-1},
\end{aligned}
$$

while the sink term for $q_{r}$ is clearly just the negative of Eq. (A16). Rain is formed at temperatures above $273.16 \mathrm{~K}$; that is, snow is assumed to melt immediately in such collisions. The source term for $q_{r}$ in this case can be found from

$$
\begin{aligned}
\left(\frac{\partial q_{r}}{\partial t}\right)_{\mathrm{AGG} ., r-s}= & \frac{\pi}{4}\left|V_{T, r}-V_{T, s}\right| N_{0, s} N_{0, r} a_{s} E_{s r}\left[\frac{2 \Gamma\left(b_{s}+1\right)}{\lambda_{r}^{3} \lambda_{s}^{b_{s}+1}}\right. \\
& \left.+\frac{2 \Gamma\left(b_{s}+2\right)}{\lambda_{r}^{2} \lambda_{s}^{b_{s}+2}}+\frac{\Gamma\left(b_{s}+3\right)}{\lambda_{r} \lambda_{s}^{b_{s}+3}}\right] \rho^{-1}
\end{aligned}
$$

and the sink term for $q_{s}$ is simply the negative of Eq. (A17). Again, the use of these source terms is similar to 
the use of PSACR and PRACS in Rutledge and Hobbs (1984) when no graupel is assumed to form.

The sink of $q_{r}$ due to evaporation is computed from

$$
\left(\frac{\partial q_{r}}{\partial t}\right)_{\mathrm{EVP}}=2 \pi\left(S_{l}-1\right)\left[0.78+0.27 \mathrm{Re}_{r}^{1 / 2}\right] G_{l}(T) N_{0, r} \lambda_{r}^{-2}
$$

when $S_{l}<1$. The quantity in square brackets is a ventilation factor, and $\mathrm{Re}_{r}$ is the Reynolds number of the average raindrop. A similar equation gives the source (sink) of $q_{s}$ due to deposition (sublimation),

$$
\left(\frac{\partial q_{s}}{\partial t}\right)_{\mathrm{DEP} / \mathrm{SUB}}=\frac{4}{3} \pi\left(S_{i}-1\right)\left[0.65+0.39 \mathrm{Re}_{s}^{1 / 2}\right] G_{i}(T) N_{0, s} \lambda_{s}^{-2},
$$

depending on whether $S_{i}$ is greater or less than unity. Again, the ventilation factor is given by the quantity in square brackets and $\mathrm{Re}_{s}$ is the Reynolds number of the average snow particle.

At temperatures exceeding $273.16 \mathrm{~K}$, snow begins to melt forming rain. The parameterization of melting rates is based on Rutledge and Hobbs (1983) resulting in a sink term for $q_{s}$,

$$
\begin{aligned}
& \left(\frac{\partial q_{s}}{\partial t}\right)_{\text {MELT }} \\
& \quad=-2 \pi K_{a} L_{f}^{-1}(T-273.16)\left[0.65+0.39 \mathrm{Re}_{s}^{1 / 2}\right] \lambda_{s}^{-2}
\end{aligned}
$$

and corresponding source term for $q_{r}$. The thermal conductivity of air is $K_{a}=2.43 \times 10^{-2} \mathrm{~J} \mathrm{~m}^{-1} \mathrm{~s}^{-1} \mathrm{~K}^{-1}$ (Rutledge and Hobbs 1983).

\section{APPENDIX B}

\section{Mixed-Phase Saturation Adjustment Procedure}

A saturation adjustment procedure is used to obtain values of temperature $T$, cloud liquid mixing ratio $q_{l}$, and cloud ice mixing ratio $q_{i}$, consistent with prognosed values of liquid-ice potential temperature $\theta_{\mathrm{li}}$, total water mixing ratio $q_{t}$, rain mixing ratio $q_{r}$, and snow mixing ratio $q_{s}$. This is done in JPLLES using a NewtonRaphson method to solve the equation

$$
f=\theta_{\mathrm{li}}-\theta \exp \left(-\frac{L_{v} q_{l, \mathrm{tot}}}{c_{p} T}-\frac{L_{s} q_{i, \mathrm{tot}}}{c_{p} T}\right)=0,
$$

where we have used the definition of $\theta_{\text {li }}$ given by Eq. (3). The latent heats are assumed to be treated as constants and saturation vapor pressures are assumed to be obtained from approximating functions rather than from direct integration of the Clausius-Clapeyron equation. The derivative of $f$ with respect to $T, f^{\prime}$, is required. This can be derived by rewriting Eq. (B1) to more clearly expose its temperature dependence:

$$
\begin{aligned}
f= & \theta_{\mathrm{li}}-\frac{T}{\Pi} \exp \left\{-\frac{L_{v} f_{l}(T) q_{c}(T)}{c_{p} T}-\frac{L_{s}\left[1-f_{l}(T)\right] q_{c}(T)}{c_{p} T}\right. \\
& \left.-\frac{L_{v} q_{r}}{c_{p} T}-\frac{L_{s} q_{s}}{c_{p} T}\right\}=0 .
\end{aligned}
$$

Here, $\Pi$ is the Exner function, $f_{l}$ is the liquid fraction determined according to Eq. (1), and $q_{c}$ is the total condensate mixing ratio given by

$$
\begin{aligned}
q_{c}(T)= & \max \left\{q_{t}-\left(q_{r}+q_{s}\right)-f_{l}(T) q_{v, \text { sat }, \text { liquid }}\right. \\
& \left.-\left[1-f_{l}(T)\right] q_{v \text {,sat,ice }}, 0\right\}
\end{aligned}
$$

Differentiation of Eq. (B2) to obtain $f^{\prime}$ is tedious but straightforward.

Once the Newton-Raphson method converges to yield a final value of $T$ (we use an absolute convergence criterion of $\left.1 \times 10^{-4} \mathrm{~K}\right), q_{i}$ and $q_{l}$ are obtained from

$$
\begin{aligned}
& q_{l}=f_{l}(T) q_{c}(T) \quad \text { and } \\
& q_{i}=\left[1-f_{l}(T)\right] q_{c}(T) .
\end{aligned}
$$

\section{APPENDIX C}

\section{Additional Results from Baseline Simulations}

Here we present selected additional results from the baseline simulations described in section $4 a$.

Figure $\mathrm{C} 1$ shows various components of the surface energy budget: downwelling longwave and shortwave radiative fluxes and the surface precipitation rate. Note that in all three cases, surface latent and sensible heat values are prescribed per the specifications of each intercomparison study and no surface energy balance is enforced. Figure $\mathrm{Cla}$ depicts the downward surface longwave radiative fluxes. The differences in our simulated values and the values obtained from observations or other models can be largely explained by their differences in liquid water path. The observed average downward surface longwave flux during MPACE was $280 \mathrm{~W} \mathrm{~m}^{-2}$ (Klein et al. 2009), thus agreeing well with the baseline simulation results through the 12-h-long observational period. Observed values of downward 

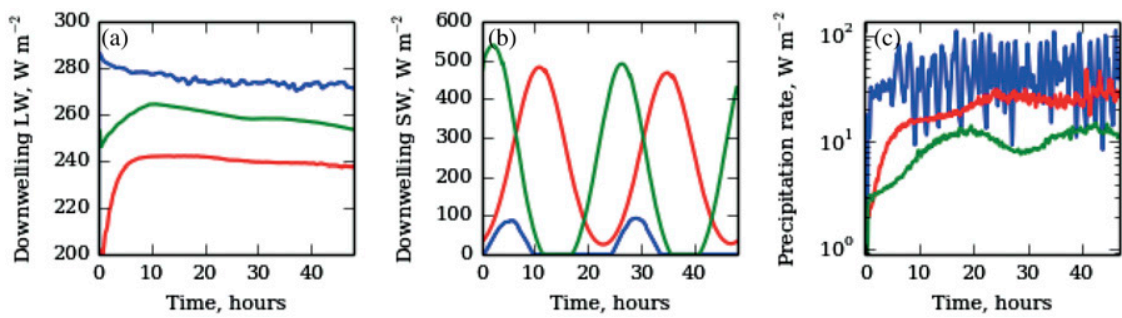

FIG. C1. Results from baseline simulations of MPACE (blue), SHEBA (red), and ISDAC (green) cases described in section 4a. Downwelling surface (a) longwave radiative flux and (b) shortwave radiative flux. (c) Surface precipitation rate, where the mass of snow has been multiplied by the latent heat of sublimation to obtain an energy flux. All quantities in $\mathrm{W} \mathrm{m}^{-2}$.

surface longwave flux during the SHEBA observational period varied between 210 and $240 \mathrm{~W} \mathrm{~m}^{-2}$, in accord with the changing LWP. The sensitivity to LWP was found to saturate for LWP $>30 \mathrm{~g} \mathrm{~m}^{-2}$ (Shupe and Intrieri 2004). Therefore, the value of approximately
$240 \mathrm{~W} \mathrm{~m}^{-2}$ attained by the downward surface longwave flux after the LWP exceeds $30 \mathrm{~g} \mathrm{~m}^{-2}$ is consistent with observations, even if the time history is not captured. Downwelling longwave fluxes on the surface during ISDAC varied between $180-250 \mathrm{~W} \mathrm{~m}^{-2}$, with a typical
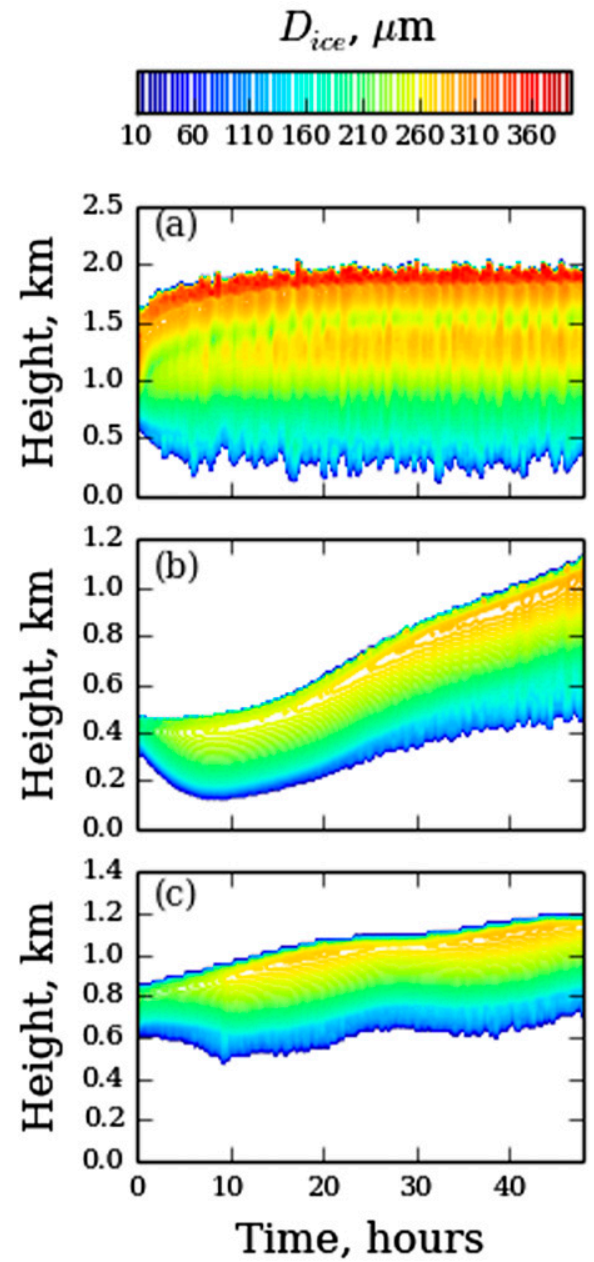
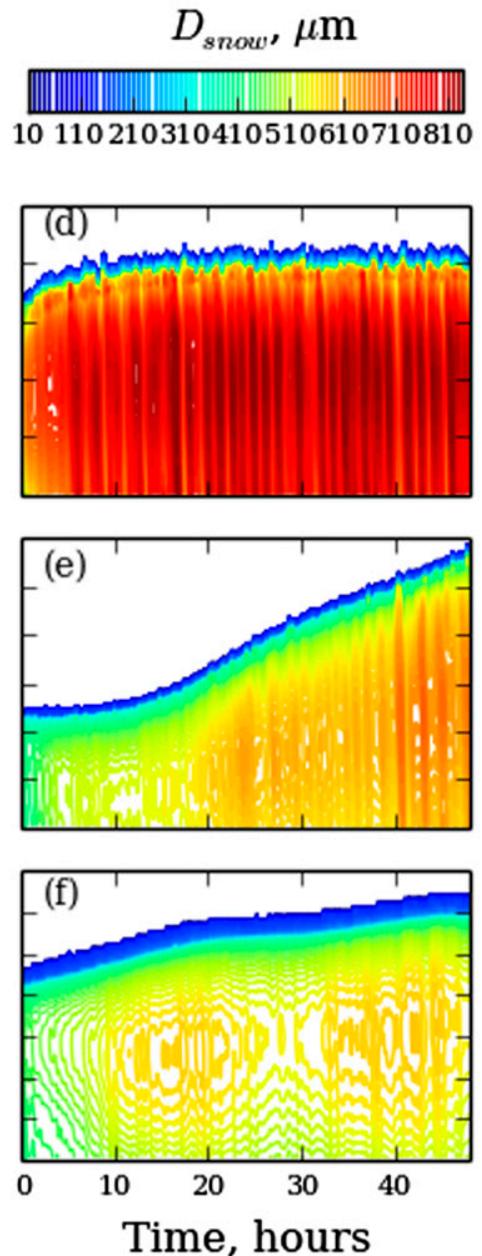
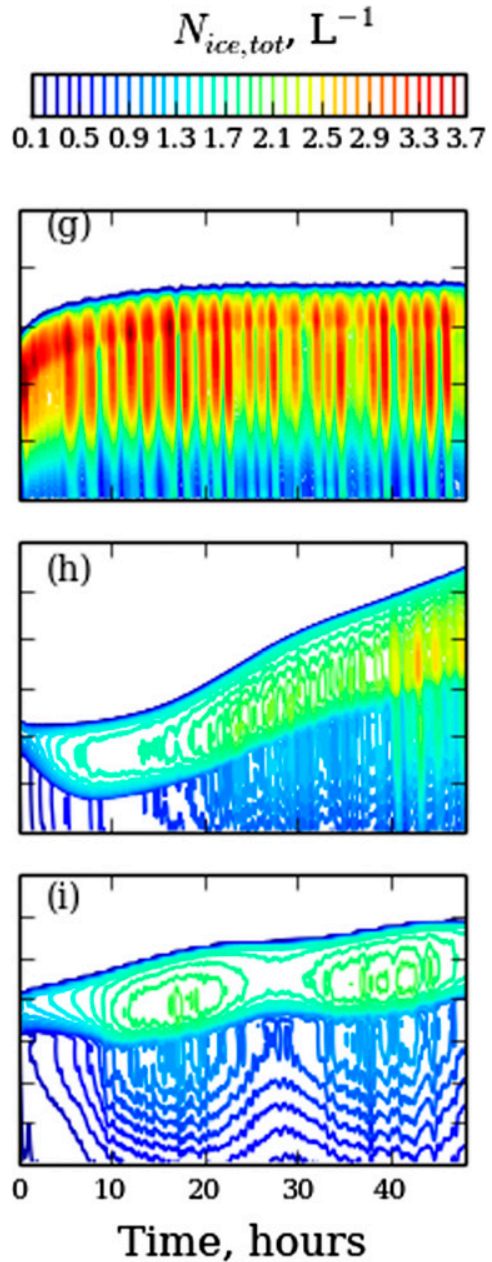

FIG. C2. Results from baseline simulations of (top) MPACE, (middle) SHEBA, and (bottom) ISDAC described in section 4a. Profiles of average (a)-(c) cloud ice particle diameter $(\mu \mathrm{m})$, (d)-(f) snow particle diameter $(\mu \mathrm{m})$, and (g)-(i) total concentration of ice particles (including cloud ice and snow) in $\mathrm{L}^{-1}$. 
value close to $200 \mathrm{~W} \mathrm{~m}^{-2}$ (Liu et al. 2011). The values obtained from the baseline simulation are higher, consistent with the higher LWP predicted by the simulation. Figure $\mathrm{C} 1 \mathrm{~b}$ depicts the downwelling shortwave flux at the surface. For both the MPACE and ISDAC cases, radiative flux observations were made from land-based instrumentation. Therefore, comparisons between the simulated and measured shortwave fluxes are impeded by the difference between the surface albedos assumed in the simulations and the surface albedo at the measurement site. The shortwave flux in the SHEBA simulation follows the correct temporal trend, but the peak value (occurring at hour 11 of the 12-h simulation period) is $30 \mathrm{~W} \mathrm{~m}^{-2}$ lower in the simulation compared to observations (Morrison et al. 2011b), consistent with the simulation's higher LWP.

The surface precipitation rate is plotted in Fig. C1c. Only the contribution from snowfall is shown as surface rainfall was either entirely absent or negligibly small in all cases. Precipitation rates are given here in units of $\mathrm{W} \mathrm{m}^{-2}$; values reported elsewhere using other units (e.g., mm day ${ }^{-1}$ liquid equivalent) are converted as needed. The median surface precipitation rate over hours 4-12 of the MPACE simulation is $35 \mathrm{Wm}^{-2}$, compared to median values of 29 and $17 \mathrm{~W} \mathrm{~m}^{-2}$ for participating single column models and cloud-resolving models (including LES models) in the intercomparison ensemble (Klein et al. 2009). The observed surface snowfall rate is highly uncertain. The measured value over the period was approximately $10 \mathrm{~W} \mathrm{~m}^{-2}$; however, a value of about $37 \mathrm{~W} \mathrm{~m}^{-2}$ is more consistent with observed near-surface ice water mixing ratios (Klein et al. 2009). All models in the SHEBA intercomparison predicted surface precipitation rates of less than $45 \mathrm{~W} \mathrm{~m}^{-2}$, following an initial ramping up period. In comparison, for all but the first hour of the observational period, measured precipitation rates ranged between 15 and $25 \mathrm{~W} \mathrm{~m}^{-2}$. Our baseline SHEBA simulation predicts an average surface precipitation rate of $14 \mathrm{~W} \mathrm{~m}^{-2}$ (averaging over hours 4-12 of the simulation), putting it close to the low end of observed values.

Figure $\mathrm{C} 2$ depicts the vertical and temporal variation in some microphysical characteristics of the simulated clouds. The ISDAC and SHEBA simulations show similar ice crystal concentrations and sizes, while the MPACE baseline simulation predicts higher concentrations and particle sizes. In particular, it should be remarked that average cloud ice crystal sizes are larger than is consistent with the assumed ice particle shape and density and zero sedimentation velocity. This could be rectified by adjusting the model parameters $n$ and $N_{0, i}$ (also see section $4 \mathrm{~d}$ ), separately or in combination with modifications to the assumed ice particle properties. However, given that the cloud ice mixing ratio is determined diagnostically and that cloud ice particle number density depends on an assumed value of $N_{0, i}$, it must be acknowledged that use of the scheme to predict characteristics of the cloud ice PSDF, which depends on both these quantities, is likely tenuous.

\section{REFERENCES}

Abel, S., and I. Boutle, 2012: An improved representation of the raindrop size distribution for single-moment microphysics schemes. Quart. J. Roy. Meteor. Soc., 138, 21512162, doi:10.1002/qj.1949.

Ackerman, A. S., and Coauthors, 2009: Large-eddy simulations of a drizzling, stratocumulus-topped marine boundary layer. Mon. Wea. Rev., 137, 1083-1110, doi:10.1175/2008MWR2582.1.

Avramov, A., and J. Harrington, 2010: Influence of parameterized ice habit on simulated mixed phase Arctic clouds. J. Geophys. Res., 115, D03205, doi:10.1029/2009JD012108.

— mixed-phase boundary layer clouds during ISDAC. J. Geophys. Res., 116, D00T08, doi:10.1029/2011JD015910.

Beare, R. J., and Coauthors, 2006: An intercomparison of largeeddy simulations of the stable boundary layer. Bound.-Layer Meteor., 118, 247-272, doi:10.1007/s10546-004-2820-6.

Berry, E. X., and R. L. Reinhardt, 1974: An analysis of cloud drop growth by collection: Part II. Single initial distributions. J. Atmos. Sci., 31, 1825-1831, doi:10.1175/1520-0469(1974)031<1825: $\mathrm{AAOCDG}>2.0 . \mathrm{CO} ; 2$.

Boudala, F., G. Isaac, S. Cober, and Q. Fu, 2004: Liquid fraction in stratiform mixed-phase clouds from in situ observations. Quart. J. Roy. Meteor. Soc., 130, 2919-2931, doi:10.1256/qj.03.153.

Boville, B., P. Rasch, J. Hack, and J. McCaa, 2006: Representation of clouds and precipitation processes in the Community Atmosphere Model Version 3 (CAM3). J. Climate, 19, 2184-2198, doi:10.1175/JCLI3749.1.

Cantrell, W., and A. Heymsfield, 2005: Production of ice in tropospheric clouds: A review. Bull. Amer. Meteor. Soc., 86, 795807, doi:10.1175/BAMS-86-6-795.

Cober, S., G. Isaac, A. Korolev, and J. Strapp, 2001: Assessing cloud-phase conditions. J. Appl. Meteor., 40, 1967-1983, doi:10.1175/1520-0450(2001)040<1967:ACPC>2.0.CO;2.

Curry, J. A., J. L. Schramm, W. B. Rossow, and D. Randall, 1996: Overview of Arctic cloud and radiation characteristics. J. Climate, 9, 1731-1764, doi:10.1175/1520-0442(1996)009<1731: OOACAR $>2.0 . \mathrm{CO} ; 2$.

Curry, J., and Coauthors, 2000: FIRE Arctic Clouds Experiment. Bull. Amer. Meteor. Soc., 81, 5-29, doi:10.1175/ 1520-0477(2000)081<0005:FACE > 2.3.CO;2.

Deardorff, J. W., 1980: Stratocumulus-capped mixed layers derived from a three-dimensional model. Bound.-Layer Meteor., 18, 495-527, doi:10.1007/BF00119502.

de Boer, G., E. W. Eloranta, and M. D. Shupe, 2009: Arctic mixedphase stratiform cloud properties from multiple years of surface-based measurements at two high-latitude locations. J. Atmos. Sci., 66, 2874-2887, doi:10.1175/2009JAS3029.1.

Devasthale, A., and M. Thomas, 2012: Sensitivity of cloud liquid water content estimates to the temperature-dependent thermodynamic phase: A global study using CloudSat data. J. Climate, 25, 7297-7307, doi:10.1175/JCLI-D-11-00521.1. 
Doutriaux-Boucher, M., and J. Quaas, 2004: Evaluation of cloud thermodynamic phase parameterizations in the LMDZ GCM by using POLDER satellite data. Geophys. Res. Lett., 31, L06126, doi:10.1029/2003GL019095.

Ervens, B., G. Feingold, K. Sulia, and J. Harrington, 2011: The impact of microphysical parameters, ice nucleation mode, and habit growth on the ice/liquid partitioning in mixed-phase Arctic clouds. J. Geophys. Res., 116, D17205, doi:10.1029/2011JD015729.

Fan, J., S. Ghan, M. Ovchinnikov, X. Liu, P. J. Rasch, and A. Korolev, 2011: Representation of Arctic mixed-phase clouds and the Wegener-Bergeron-Findeisen process in climate models: Perspectives from a cloud-resolving study. J. Geophys. Res., 116, D00T07, doi:10.1029/2010JD015375.

Fridlind, A., A. Ackerman, G. McFarquhar, G. Zhang, M. Poellot, P. DeMott, A. Prenni, and A. Heymsfield, 2007: Ice properties of single-layer stratocumulus during the Mixed-Phase Arctic Cloud Experiment: 2. Model results. J. Geophys. Res., 112 D24202, doi:10.1029/2007JD008646.

- B. van Diedenhoven, A. Ackerman, A. Avramov, A. Mrowiec, H. Morrison, P. Zuidema, and M. Shupe, 2012: A FIRE-ACE/SHEBA case study of mixed-phase Arctic boundary layer clouds: Entrainment rate limitations on rapid primary ice nucleation processes. J. Atmos. Sci., 69, 365-389, doi:10.1175/JAS-D-11-052.1.

Gilmore, M., and J. Straka, 2008: The Berry and Reinhardt autoconversion parameterization: A digest. J. Appl. Meteor. Climatol., 47, 375-396, doi:10.1175/2007JAMC1573.1.

Grabowski, W., 1989: On the influence of small-scale topography on precipitation. Quart. J. Roy. Meteor. Soc., 115, 633-650, doi:10.1002/qj.49711548711.

—_ 1998: Toward cloud resolving modeling of large-scale tropical circulations: A simple cloud microphysics parameterization. J. Atmos. Sci., 55, 3283-3298, doi:10.1175/ 1520-0469(1998)055<3283:TCRMOL > 2.0.CO;2.

Gregory, D., and D. Morris, 1996: The sensitivity of climate simulations to the specification of mixed phase clouds. Climate Dyn., 12, 641-651, doi:10.1007/BF00216271.

Harrington, J. Y., M. P. Meyers, R. L. Walko, and W. R. Cotton, 1995: Parameterization of ice crystal conversion processes due to vapor deposition for mesoscale models using doublemoment basis functions. Part I: Basic formulation and parcel model results. J. Atmos. Sci., 52, 4344-4366, doi:10.1175/ 1520-0469(1995)052<4344:POICCP >2.0.CO;2.

—, T. Reisin, W. R. Cotton, and S. M. Kreidenweis, 1999: Cloud resolving simulations of Arctic stratus: Part II: Transition-season clouds. Atmos. Res., 51, 45-75, doi:10.1016/ S0169-8095(98)00098-2.

Held, I. M., 2005: The gap between simulation and understanding in climate modeling. Bull. Amer. Meteor. Soc., 86, 1609-1614, doi:10.1175/BAMS-86-11-1609.

Heymsfield, A. J., A. Bansemer, and C. H. Twohy, 2007: Refinements to ice particle mass dimensional and terminal velocity relationships for ice clouds. Part I: Temperature dependence. J. Atmos. Sci., 64, 1047-1067, doi:10.1175/ JAS3890.1.

Hill, A. A., P. R. Field, K. Furtado, A. Korolev, and B. J. Shipway, 2014: Mixed-phase clouds in a turbulent environment. Part 1: Large-eddy simulation experiments. Quart. J. Roy. Meteor. Soc., 140, 855-869, doi:10.1002/qj.2177.

Intrieri, J., C. Fairall, M. Shupe, P. Persson, E. L Andreas, P. Guest, and R. Moritz, 2002: An annual cycle of Arctic surface cloud forcing at SHEBA. J. Geophys. Res., 107, 8039, doi:10.1029/ 2000JC000439.
Jiang, G.-S., and D. Peng, 2000: Weighted ENO schemes for Hamilton-Jacobi equations. SIAM J. Sci. Comput., 21, 21262143, doi:10.1137/S106482759732455X.

Jiang, H., W. Cotton, J. Pinto, J. Curry, and M. Weissbluth, 2000: Cloud resolving simulations of mixed-phase Arctic stratus observed during BASE: Sensitivity to concentrations of ice crystals and large-scale heat and moisture advection. J. Atmos. Sci., 57, 2105-2117, doi:10.1175/1520-0469(2000)057<2105: $\mathrm{CRSOMP}>2.0 . \mathrm{CO} ; 2$

Kay, J., and A. Gettelman, 2009: Cloud influence on and response to seasonal Arctic sea ice loss. J. Geophys. Res., 114, D18204, doi:10.1029/2009JD011773.

Khairoutdinov, M., and D. Randall, 2003: Cloud resolving modeling of the ARM summer 1997 IOP: Model formulation, results, uncertainties, and sensitivities. J. Atmos. Sci., 60, 607-625, doi:10.1175/1520-0469(2003)060<0607: CRMOTA $>2.0 . \mathrm{CO} ; 2$

Klein, S., and Coauthors, 2009: Intercomparison of model simulations of mixed-phase clouds observed during the ARM MixedPhase Arctic Cloud Experiment. I: Single-layer cloud. Quart. J. Roy. Meteor. Soc., 135, 979-1002, doi:10.1002/qj.416.

Klemp, J., and R. Wilhemson, 1978: The simulation of threedimensional convective storm dynamics. J. Atmos. Sci., 35, 1070-1096, doi:10.1175/1520-0469(1978)035<1070: TSOTDC $>2.0 . \mathrm{CO} ; 2$

Korolev, A. V., 2008: Rates of phase transformation in mixedphase clouds. Quart. J. Roy. Meteor. Soc., 134, 595-608, doi:10.1002/qj.230.

and G. Isaac, 2003: Phase transformation of mixed-phase clouds. Quart. J. Roy. Meteor. Soc., 129, 19-38, doi:10.1256/ qj.01.203

- — - S. G. Cober, J. W. Strapp, and J. Hallett, 2003: Microphysical characterization of mixed-phase clouds. Quart. J. Roy. Meteor. Soc., 129, 39-65, doi:10.1256/qj.01.204.

Li, Z.-X., and H. L. Treut, 1992: Cloud-radiation feedbacks in a general circulation model and their dependence on cloud modelling assumptions. Climate Dyn., 7, 133-139, doi:10.1007/ BF00211155.

Liu, X., and Coauthors, 2011: Testing cloud microphysics parameterizations in NCAR CAM5 with ISDAC and M-PACE observations. J. Geophys. Res., 116, D00T11, doi:10.1029/ 2011JD015889.

Luo, Y., K.-M. Xu, H. Morrison, and G. McFarquhar, 2008: Arctic mixed-phase clouds simulated by a cloud-resolving model: Comparison with ARM observations and sensitivity to microphysics parameterizations. J. Atmos. Sci., 65, 1285-1303, doi:10.1175/2007JAS2467.1.

Martin, G., D. Johnson, and A. Spice, 1994: The measurement and parameterization of effective radius of droplets in warm stratocumulus clouds. J. Atmos. Sci., 51, 1823-1842, doi:10.1175/1520-0469(1994)051<1823:TMAPOE $>2.0 . C O ; 2$.

Matheou, G., D. Chung, L. Nuijens, B. Stevens, and J. Teixeira, 2011: On the fidelity of large-eddy simulation of shallow precipitating cumulus convection. Mon. Wea. Rev., 139, 29182939, doi:10.1175/2011MWR3599.1.

McFarquhar, G., G. Zhang, M. Poellot, G. Kok, R. McCoy, T. Tooman, A. Fridlind, and A. Heymsfield, 2007: Ice properties of single-layer stratocumulus during the Mixed-Phase Arctic Cloud Experiment: 1. Observations. J. Geophys. Res., 112, D24201, doi:10.1029/2007JD008633.

and Coauthors, 2011: Indirect and Semi-Direct Aerosol Campaign: The impact of Arctic aerosols on clouds. Bull. Amer. Meteor. Soc., 92, 183-201, doi:10.1175/2010BAMS2935.1. 
Morrison, H., and W. W. Grabowski, 2008: A novel approach for representing ice microphysics in models: Description and tests using a kinematic framework. J. Atmos. Sci., 65, 1528-1548, doi:10.1175/2007JAS2491.1.

—, M. D. Shupe, J. O. Pinto, and J. A. Curry, 2005: Possible roles of ice nucleation mode and ice nuclei depletion in the extended lifetimes of Arctic mixed-phase clouds. Geophys. Res. Lett., 32, L18801, doi:10.1029/2005GL023614.

—, P. Zuidema, G. M. McFarquhar, A. Bansemer, and A. J. Heymsfield, 2011a: Snow microphysical observations in shallow mixed-phase and deep frontal Arctic cloud systems. Quart. J. Roy. Meteor. Soc., 137, 1589-1601, doi:10.1002/ qj. 840 .

— , and Coauthors, 2011b: Intercomparison of cloud model simulations of Arctic mixed-phase boundary layer clouds observed during SHEBA/FIRE-ACE. J. Adv. Model. Earth Syst., 3, M05001, doi:10.1029/2011MS000066.

—, G. de Boer, G. Feingold, J. Harrington, M. Shupe, and K. Sulia, 2012: Resilience of persistent Arctic mixed-phase clouds. Nat. Geosci., 5, 11-17, doi:10.1038/ngeo1332.

Noh, Y.-J., C. Seaman, T. H. Vonder Haar, and G. Liu, 2013: In situ aircraft measurements of the vertical distribution of liquid and ice water content in midlatitude mixed-phase clouds. J. Appl. Meteor. Climatol., 52, 269-279, doi:10.1175/ JAMC-D-11-0202.1.

Ovchinnikov, M., A. Korolev, and J. Fan, 2011: Effects of ice number concentration on dynamics of a shallow mixed-phase stratiform cloud. J. Geophys. Res., 116, D00T06, doi:10.1029/ 2011JD015888.

—_, and Coauthors, 2014: Intercomparison of large-eddy simulations of Arctic mixed-phase clouds: Importance of ice size distribution assumptions. J. Adv. Model. Earth Syst., 6, 223 248, doi:10.1002/2013MS000282.

Pruppacher, H. R., and J. D. Klett, 1978: Microphysics of Clouds and Precipitation. D. Reidel, $954 \mathrm{pp}$.

Rauber, R., and A. Tokay, 1991: An explanation for the existence of supercooled water at the top of cold clouds. J. Atmos. Sci., 48, 1005-1023, doi:10.1175/1520-0469(1991)048<1005: AEFTEO $>2.0 . \mathrm{CO} ; 2$.

Reisner, J., R. Rasmussen, and R. Bruintjes, 1998: Explicit forecasting of supercooled liquid water in winter storms using the MM5 mesoscale model. Quart. J. Roy. Meteor. Soc., 124, 10711107, doi:10.1002/qj.49712454804.

Rutledge, S., and P. Hobbs, 1983: The mesoscale and microscale structure and organization of clouds and precipitation in midlatitude cyclones. VIII: A model for the "seederfeeder" process in warm-frontal rainbands. J. Atmos. Sci., 40, 1185-1206, doi:10.1175/1520-0469(1983)040<1185: TMAMSA $>2.0 . \mathrm{CO} ; 2$.

, and - 1984: The mesoscale and microscale structure and organization of clouds and precipitation in midlatitude cyclones. XII: A diagnostic modeling study of precipitation development in narrow cold-frontal bands. J. Atmos. Sci., 41, 2949-2972, doi:10.1175/1520-0469(1984)041<2949: TMAMSA $>2.0 . \mathrm{CO} ; 2$.

Savre, J., A. M. L. Ekman, and G. Svensson, 2014: Technical note: Introduction to MIMICA, a large-eddy simulation solver for cloudy planetary boundary layers. J. Adv. Model. Earth Syst., 6, 630-649, doi:10.1002/2013MS000292.

Seifert, A., and K. D. Beheng, 2006: A two-moment cloud microphysics parameterization for mixed-phase clouds. Part 1: Model description. Meteor. Atmos. Phys., 92, 45-66, doi:10.1007/ s00703-005-0112-4.
Sekhon, R. S., and R. C. Srivastava, 1970: Snow size and radar reflectivity. J. Atmos. Sci., 27, 299-307, doi:10.1175/ 1520-0469(1970)027<0299:SSSARR>2.0.CO;2.

Shupe, M., 2011: Clouds at Arctic atmospheric observatories. Part II: Thermodynamic phase characteristics. J. Appl. Meteor. Climatol., 50, 645-661, doi:10.1175/2010JAMC2468.1.

— surface: The influence of cloud properties, surface albedo, and solar zenith angle. J. Climate, 17, 616-628, doi:10.1175/ 1520-0442(2004)017<0616:CRFOTA > 2.0.CO;2.

— S. Matrosov, and T. Uttal, 2006: Arctic mixed-phase cloud properties derived from surface-based sensors at SHEBA. J. Atmos. Sci., 63, 697-711, doi:10.1175/JAS3659.1.

— , P. Kollias, P. Persson, and G. McFarquhar, 2008: Vertical motions in Arctic mixed-phase stratiform clouds. J. Atmos. Sci., 65, 1304-1332, doi:10.1175/2007JAS2479.1.

Solomon, A., H. Morrison, O. Persson, M. Shupe, and J.-W. Bao, 2009: Investigation of microphysical parameterizations of snow and ice in Arctic clouds during M-PACE through model-observation comparisons. Mon. Wea. Rev., 137, 3110 3128, doi:10.1175/2009MWR2688.1.

_- M. Shupe, P. Persson, and H. Morrison, 2011: Moisture and dynamical interactions maintaining decoupled Arctic mixedphase stratocumulus in the presence of a humidity inversion. Atmos. Chem. Phys., 11, 10127-10148, doi:10.5194/ acp-11-10127-2011.

— - - O. Persson, H. Morrison, T. Yamaguchi, P. M. Caldwell, and G. de Boer, 2014: The sensitivity of springtime Arctic mixed-phase stratocumulus clouds to surface layer and cloud-top inversion layer moisture sources. J. Atmos. Sci., $\mathbf{7 1}$, 574-595, doi:10.1175/JAS-D-13-0179.1.

Spalart, P. R., R. D. Moser, and M. M. Rogers, 1991: Spectral methods for the Navier-Stokes equations with one infinite and two periodic dimensions. J. Comput. Phys., 96, 297-324, doi:10.1016/0021-9991(91)90238-G.

Stephens, G., P. Gabriel, and P. Partain, 2001: Parameterization of atmospheric radiative transfer. Part I: Validity of simple models. J. Atmos. Sci., 58, 3391-3409, doi:10.1175/ 1520-0469(2001)058<3391:POARTP > 2.0.CO;2.

Stevens, B., and Coauthors, 2005: Evaluation of large-eddy simulations via observations of nocturnal marine stratocumulus. Mon. Wea. Rev., 133, 1443-1462, doi:10.1175/MWR2930.1.

Sulia, K. J., and J. Y. Harrington, 2011: Ice aspect ratio influences on mixed-phase clouds: Impacts on phase partitioning in parcel models. J. Geophys. Res., 116, D21309, doi:10.1029/ 2011JD016298.

Thompson, G., R. Rasmussen, and K. Manning, 2004: Explicit forecasts of winter precipitation using an improved bulk microphysical scheme. Part I: Description and sensitivity analysis. Mon. Wea. Rev., 132, 519-542, doi:10.1175/ 1520-0493(2004)132<0519:EFOWPU>2.0.CO;2.

-, P. Field, R. Rasmussen, and W. Hall, 2008: Explicit forecasts of winter precipitation using an improved bulk microphysical scheme. Part II: Implementation of a new snow parameterization. Mon. Wea. Rev., 136, 5095-5115, doi:10.1175/ 2008MWR2387.1.

Tripoli, G. J., and W. R. Cotton, 1981: The use of ice-liquid water potential temperature as a thermodynamic variable in deep atmospheric models. Mon. Wea. Rev., 109, 1094-1102, doi:10.1175/1520-0493(1981)109<1094:TUOLLW>2.0.CO;2.

Uttal, T., and Coauthors, 2002: Surface heat budget of the Arctic Ocean. Bull. Amer. Meteor. Soc., 83, 255-275, doi:10.1175/ 1520-0477(2002)083<0255:SHBOTA > 2.3.CO;2. 
van Diedenhoven, B., A. M. Fridlind, A. S. Ackerman, E. W. Eloranta, and G. M. McFarquhar, 2009: An evaluation of ice formation in large-eddy simulations of supercooled arctic stratocumulus using ground-based lidar and cloud radar. J. Geophys. Res., 114, D10203, doi:10.1029/ 2008JD011198.

Vavrus, S., 2004: The impact of cloud feedbacks on Arctic climate under greenhouse forcing. J. Climate, 17, 603-615, doi:10.1175/ 1520-0442(2004)017<0603:TIOCFO >2.0.CO;2.

- U. Bhatt, and V. Alexeev, 2011: Factors influencing simulated changes in future Arctic cloudiness. J. Climate, 24, 48174830, doi:10.1175/2011JCLI4029.1.
Verlinde, J., and Coauthors, 2007: The Mixed-Phase Arctic Cloud Experiment. Bull. Amer. Meteor. Soc., 88, 205-221, doi:10.1175/ BAMS-88-2-205.

Wood, R., 2012: Stratocumulus clouds. Mon. Wea. Rev., 140, 2373 2423, doi:10.1175/MWR-D-11-00121.1.

Yang, F., M. Ovchinnikov, and R. Shaw, 2013: Minimalist model of ice microphysics in mixed-phase stratiform clouds. Geophys. Res. Lett., 40, 3756-3760, doi:10.1002/ grl.50700.

Zuidema, P., and Coauthors, 2005: An Arctic springtime mixedphase cloudy boundary layer observed during SHEBA. J. Atmos. Sci., 62, 160-176, doi:10.1175/JAS-3368.1. 